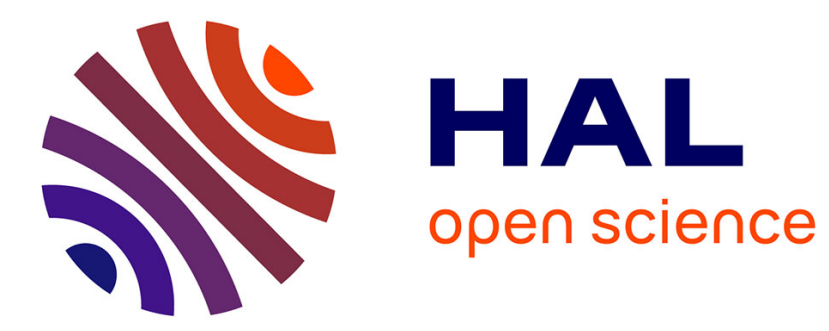

\title{
Modeling flow in porous media with rough surfaces: effective slip boundary conditions and application to structured packings
}

\author{
Sylvain Pasquier, Michel Quintard, Yohan Davit
}

\section{To cite this version:}

Sylvain Pasquier, Michel Quintard, Yohan Davit. Modeling flow in porous media with rough surfaces: effective slip boundary conditions and application to structured packings. Chemical Engineering Science, 2017, vol. 165, pp. 131-146. 10.1016/j.ces.2017.01.063 . hal-01494265

\section{HAL Id: hal-01494265 \\ https://hal.science/hal-01494265}

Submitted on 23 Mar 2017

HAL is a multi-disciplinary open access archive for the deposit and dissemination of scientific research documents, whether they are published or not. The documents may come from teaching and research institutions in France or abroad, or from public or private research centers.
L'archive ouverte pluridisciplinaire HAL, est destinée au dépôt et à la diffusion de documents scientifiques de niveau recherche, publiés ou non, émanant des établissements d'enseignement et de recherche français ou étrangers, des laboratoires publics ou privés. 


\section{OATAO \\ Open Archive Toulouse Archive Ouverte}

\section{Open Archive TOULOUSE Archive Ouverte (OATAO)}

OATAO is an open access repository that collects the work of Toulouse researchers and makes it freely available over the web where possible.

This is an author-deposited version published in : http://oatao.univ-toulouse.fr/ Eprints ID : 17495

To link to this article : DOI:10.1016/j.ces.2017.01.063

URL : http://dx.doi.org/10.1016/j.ces.2017.01.063

To cite this version : Pasquier, Sylvain and Quintard, Michel and Davit, Yohan Modeling flow in porous media with rough surfaces: effective slip boundary conditions and application to structured packings. (2017) Chemical Engineering Science, vol. 165. pp. 131146. ISSN 0009-2509

Any correspondence concerning this service should be sent to the repository administrator: staff-oatao@,listes-diff.inp-toulouse.fr 


\title{
Modeling flow in porous media with rough surfaces: Effective slip boundary conditions and application to structured packings
}

\author{
Sylvain Pasquier, Michel Quintard, Yohan Davit* \\ Institut de Mécanique des Fluides de Toulouse (IMFT) - Université de Toulouse, CNRS-INPT-UPS, Toulouse France
}

H I G H L I G H T S

- An upscaling methodology is proposed to characterize fluid flow in porous media with rough surfaces.

- The no-slip condition on the rough surface is replaced with a tensorial slip condition on a fictive smooth surface.

- The permeability decreases significantly, even for small amplitudes of the roughness.

- The pressure drop is accurately predicted for structured packings used in separation processes.

- Different regimes are identified in the transition from creeping flow to the onset of unsteady inertial flow.

Keywords:

Porous media

Structured packings

Upscaling

Rough surface

Effective condition

\begin{abstract}
A B S T R A C T
Understanding and modeling flows in columns equipped with structured packings is crucial to enhance the efficiency of many processes in chemical engineering. As in most porous media, an important factor that affects the flow is the presence of rough surfaces, whether this roughness has been engineered as a texture on the corrugated sheets or is the result of hydrodynamic instabilities at the interface between a gas and a liquid phase. Here, we develop a homogenized model for flows in generic porous media with rough surfaces. First, we derive a tensorial form of an effective slip boundary condition that replaces the no-slip condition on the complex rough structure and captures surface anisotropy. Second, a Darcy-Forchheimer model is obtained using the volume averaging method to homogenize the porescale equations with the effective slip condition. The advantage of decomposing the upscaling in these two steps is that the effective parameters at the Darcy-scale can be calculated in a representative volume with smooth boundaries, therefore considerably simplifying mesh construction and computations. The approach is then applied to a variety of geometries, including structured packings, and compared with direct numerical solutions of the flow to evaluate its accuracy over a wide range of Reynolds number. We find that the roughness can significantly impact the flow and that this impact is accurately captured by the effective boundary condition for moderate Reynolds numbers. We further discuss the dependance of the permeability and generalized Forchheimer terms upon the Reynolds number and propose a classification into distinct regimes.
\end{abstract}

\section{Introduction}

Structured packings are widely used in industrial processes for phase separation such as distillation or post-combustion $\mathrm{CO}_{2}$ capture. These often consist of parallel corrugated sheets packed together in a way that maintains a large void fraction and surface area, therefore maximizing transfers between phases. Prediction of the pressure drop in columns equipped with such packings is a major concern for users and manufacturers alike. Recent develop-

\footnotetext{
* Corresponding author.

E-mail address: yohan.davit@imft.fr (Y. Davit).
}

ments in computational fluid dynamics (CFD), along with the advent of high performance computing, are providing the necessary basis to better understand flow in these complex structures and, ultimately, to optimize their design.

Column-scale computations of the flow at the pore-scale, however, is still not feasible. Most simulations focus on a restricted part of the domain, for instance a representative elementary volume (REV) of structured packings. Calculations over a REV are useful to understand the micro-scale physics and evaluate effective parameters that apply to the packing and the column-scale, while being small enough to allow for accurate simulations resolving the smallest scales of the flow. This strategy, inspired from approaches 


\section{Nomenclature}

Greek symbols

$\gamma \quad$ fluid phase

$\sigma \quad$ solid phase

$\rho_{\gamma} \quad$ density of the phase $\gamma$

$\mu_{\gamma} \quad$ dynamic viscosity of the phase $\gamma$

$\lambda$ wavelength of the roughness

$\mathcal{A}$ amplitude of the roughness

$\mathcal{V} \quad$ volume

$\Omega \quad$ domain

$\phi \quad$ volume fractions of the domains $\Omega$

$\Omega_{w_{i}} \quad$ small domain over a unit rough element

$\kappa \quad$ curvature of the interface $\Gamma_{w s}$

$\Gamma_{\gamma \sigma} \quad$ surface of the rough porous medium

$\Gamma_{w s} \quad$ interface between the two sub-domains $\Omega_{w}$ and $\Omega_{s}$

$\Gamma_{\gamma \sigma}^{\text {smooth }}$ surface of the smooth domain $\Omega_{\text {smooth }}$ positioned at the mean position of the roughness

\section{Roman symbols}

$l \quad$ characteristic length-scale of the pores

$l_{w} \quad$ distance between the rough wall and the effective surface
$L \quad$ characteristic length associated with the averaging domain $\Omega$

$d \quad$ diameter of the cylinders

$H \quad$ characteristic length for the 2D channel and for structured packings

$p_{\gamma}, p_{s}, p_{w}$ pressure defined respectively in $\Omega_{\gamma}, \Omega_{s}, \Omega_{w}$

$\mathbf{u}_{\gamma}, \mathbf{u}_{s}, \mathbf{u}_{w}$ fluid velocity defined respectively in the domain $\Omega_{\gamma}, \Omega_{s}, \Omega_{w}$

a, A couple of mapping variables for pressure and velocity in $\Omega_{w_{i}}$

$\mathbf{b}_{s}, \mathbf{B}_{s} \quad$ couple of mapping variables for pressure and velocity in $\Omega_{\mathrm{S}}$

F inertial correction tensors

$\mathbf{K}^{\star} \quad$ apparent permeability

$k^{\star} \quad$ dimensionless permeabilities

g gravitational acceleration

$g_{p} \quad$ global pressure gradient

$g_{s} \quad$ global dissipation rate

$R_{d} \quad$ Reynolds number with $d$ as characteristic length used in porous media sciences, was first introduced by Petre et al. (2003), who modeled the gas flow within four REVs in the viscous and turbulent regimes. Once effective parameters, such as the permeability tensor, have been evaluated in this REV, the whole column can be treated as a homogenized porous medium. For instance, momentum transport at the column-scale can be described by Darcy-Forchheimer's law, which can then be solved numerically on a coarse mesh. This approach yields results that are generally more accurate than earlier empirical correlations, such as those proposed by Brunazzi and Paglianti (1997), Olujic (1999) and Rocha et al. (1993).

A recent contribution that uses the porous media approach to study flow and pressure drop in gas-liquid distillation columns is the work by Mahr and Mewes (2008). The authors introduced a flow resistance tensor that depends on the velocity magnitude, which can be used to predict the pressure drop for any flow direction. Soulaine and Quintard (2014) further investigated anisotropy generated at the micro-scale by inertial flow and proposed an upscaling methodology to determine a correction tensor in the Darcy-Forchheimer's law. Their conclusion, for $45^{\circ}$ inclined corrugations, is that the non-diagonal terms in the effective secondorder tensors can be neglected in comparison to the diagonal ones.

In addition to inertial effects and anisotropy, an important factor that affects the gas pressure drop in these columns is the potential presence of rough surfaces. For instance, the corrugated sheets may be textured to increase the efficiency of distillation processes (McGlamery, 1988) or hydrodynamic instabilities may develop at the liquid-gas interface as a result of the steep flowing angle of the film and the high contrast in velocity between the two phases (Zapke and Kröger, 2000; Dietze and Ruyer-Quil, 2013). Those instabilities, which can manifest as soliton structures, are likely to generate dramatic changes of the pressure drop and contribute to the flooding of the column for a large flow rate of the gas phase (Trifonov, 2010). Based on the contrasts in density, viscosity and velocity between the two phases, one often assumes that the gas flow can be treated as a single-phase flow over a rigid rough surface. For this to be accurate, the velocity in the gas phase must be much larger than the velocity in the liquid phase so that we can use a no-slip boundary condition (see also (Vellingiri et al., 2013) for additional details). The trains of waves can then be treated as a surface roughness for the pressure drop in the gasphase. This is also consistent with previous works that treat the pressure drop of the gas phase a posteriori, by considering the liquid film hold-up for the calculation of the interstitial gas velocity (Raynal and Royon-Lebeaud, 2007).

Here, we propose a multi-scale approach to evaluate the impact of rough surfaces on the macro-scale properties of momentum transport. We develop a systematic methodology for a generic porous medium, independently of the initial nature of the roughness, which is based on the idea that the rough surface can be replaced by an effective boundary condition over a smooth surface, to reduce computation time and limit issues with the mesh construction. To this end, we start by describing the pore-scale system of equations in Section 2, along with the domain decomposition method leading to the derivation of an effective slip boundary condition that replaces the rough surface. We then go on to upscale these equations in Section 3 using the volume averaging with closure technique to obtain the macro-scale equations that describe momentum transport along with the closure problems that are used to calculate effective parameters. In Section 4, we apply this generic approach to a variety of geometries including a representative element of structured packings. We evaluate the accuracy of our approach and the impact of the roughness on the effective parameters. Finally, in Section 5, we discuss the limitations of the framework and provide ideas for future improvements.

\section{Derivation of an effective slip condition}

As discussed in Section 1, the goal of this paper is to develop an upscaling methodology that can be used to evaluate the impact of rough surfaces in porous media. To this end, we will use the method of volume averaging with closure, which has been widely documented since the early work of Whitaker (1986). This method usually yields a macro-scale model involving effective parameters that are computed by solving closure problems over a REV. An important implication of rough surfaces is that computations of the closure problems over a REV may not be tractable if the characteristic amplitude, $\mathcal{A}$, and wavelength, $\lambda$, of the roughnesses are small compared to the pore-scale, $l$. If this is the case, i.e. if $\frac{\mathcal{A}}{I} \ll 1$ 
and $\frac{\lambda}{l} \ll 1$, we may need an extremely fine mesh to accurately capture the flow over the roughness, especially if the Reynolds number is larger than unity. Here, we solve this issue by developing an alternative formulation of the pore-scale flow problem, transforming the rough surface into a smooth one. The no-slip condition applying to the complex rough surface is replaced by an effective boundary condition over a smooth surface, therefore limiting the need for a very fine mesh in the vicinity of the surface.

\subsection{Micro-scale transport equations}

We consider the flow of an incompressible fluid $\gamma$, within a porous structure with rough walls, as shown in Fig. 1. Mass and momentum balance are described via the Navier-Stokes equations as

$\nabla \cdot \mathbf{u}_{\gamma}=0$

$\rho_{\gamma}\left(\frac{\partial \mathbf{u}_{\gamma}}{\partial t}+\mathbf{u}_{\gamma} \cdot \nabla \mathbf{u}_{\gamma}\right)=-\nabla p_{\gamma}+\nabla \cdot\left[\mu_{\gamma}\left(\nabla \mathbf{u}_{\gamma}+\left(\nabla \mathbf{u}_{\gamma}\right)^{T}\right)\right]+\rho_{\gamma} \mathbf{g}$

$\mathbf{u}_{\gamma}=0$ on $\Gamma_{\gamma \sigma}$

where $\mathbf{u}_{\gamma}, p$ are the velocity and pressure fields in the rough domain, $\Omega_{\gamma} . \rho_{\gamma}$ and $\mu_{\gamma}$ are the density and viscosity of the phase $\gamma$.

The first step of our methodology is to replace Eqs. (1a)-(1c) in $\Omega_{\gamma}$ and on $\Gamma_{\gamma \sigma}$ by an effective mathematical system in an effective domain $\Omega_{s}$ and $\Gamma_{w s}$, where the subscript $s$ means "slip".

\subsection{Domain decomposition and effective slip}

We consider the domain $\Omega_{\gamma} \subset \mathbb{R}^{m}$, ( $m=2$ or 3 ), with the rough boundary $\Gamma_{\gamma \sigma} \subset \mathbb{R}^{m-1}$, as presented in Fig. 2 . The roughness is characterized by an amplitude, $\mathcal{A}$, and a wavelength, $\lambda$. We further use $l$ to represent a characteristic size of the pores. Similarly to the approaches of Achdou et al. (1998a,b), Veran et al. (2009) and Introini et al. (2011), we decompose the fluid domain in two subdomains: one close to the rough wall, $\Omega_{w}$, and one that represents the center of the pores, $\Omega_{s}$. Therefore, we have $\Omega_{\gamma}=\Omega_{w} \cup \Omega_{s}$ and the two subdomains are separated by the surface $\Gamma_{w s}$, as illustrated in Fig. 2. To derive an effective boundary condition applying to $\Gamma_{w s}$, Achdou et al. used a technique based on a two-scale asymptotic expansion (Achdou et al., 1998a). This method is also used in the works of Barrenechea et al. (2002), Basson and Gérard-Varet (2008), and Vincent and Andro (2002). Veran et al. and Introïni et al. used a different method based on the resolution of a local boundary problem in the small representative domain $\Omega_{w_{i}}$, also using ideas similar to those of porous media homogenization

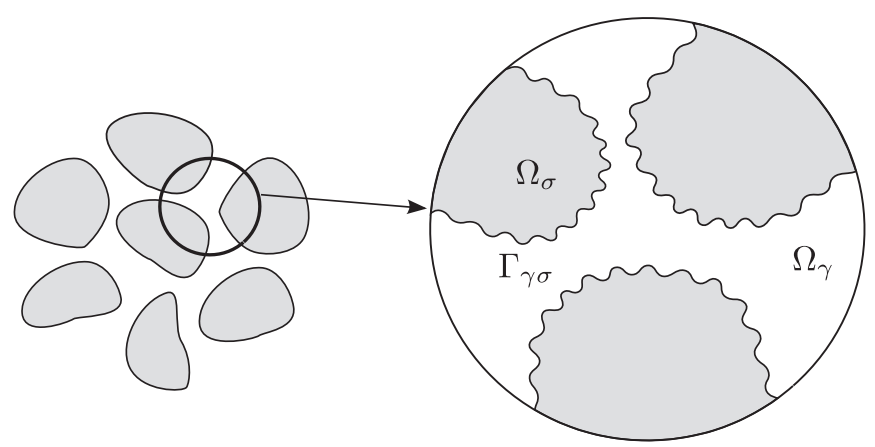

Fig. 1. Schematics of a porous medium with rough walls. $\Omega_{\sigma}$ and $\Omega_{\gamma}$ are, respectively, the solid and fluid phases. $\Gamma_{\gamma \sigma}$ is the rough surface of the porous medium.

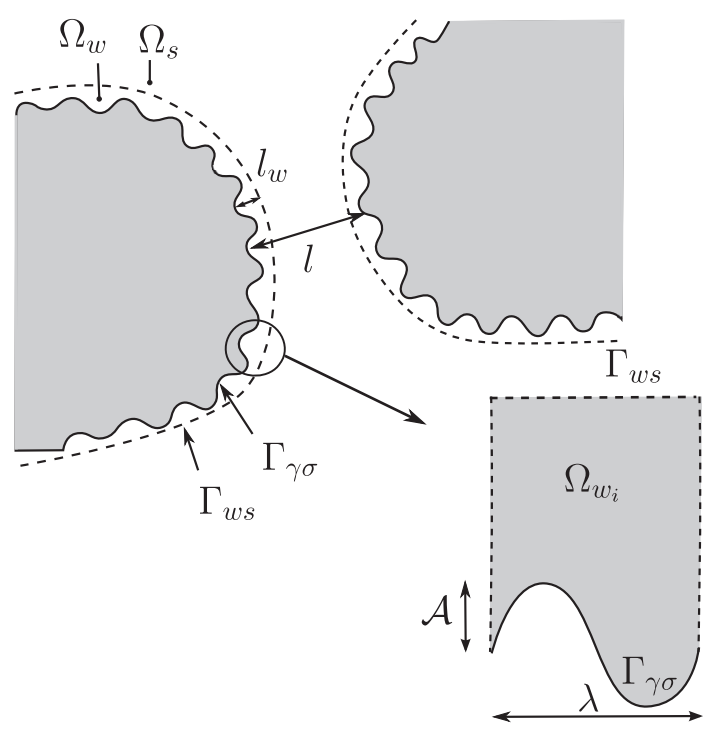

Fig. 2. Schematics of the multi-domain decomposition. $\Gamma_{\text {ws }}$ and $\Omega_{s}$ refer to the effective surface and domain, respectively. $\Omega_{w}$ is the subdomain that contains the rough wall and $\Omega_{w_{i}}$ is the local domain over a rough element. $l_{w}$ is the characteristic distance from the rough pattern to the effective surface and $l$ is the characteristic length scale of the pore. $\mathcal{A}$ and $\lambda$ are the amplitude and wavelength of the roughness.

approaches. Here, we present briefly the main ideas of this last approach with a particular focus on the tensor formalism for the slip length. More detailed developments are described in Veran et al. (2009) and Introini et al. (2011).

The main assumptions of the effective boundary condition are as follows:

- The impact of the roughness on the flow is localized to a viscous boundary layer above the roughness. This is correct when the Reynolds number is sufficiently low or in the fully turbulent regime if the roughness is smaller than the viscous sublayer (Sternberg, 1962).

- The interface $\Gamma_{w s}$ is placed sufficiently far above the roughness, so that the pressure and velocity fluctuations due to the roughness vanish on $\Gamma_{\text {ws }}$.

- The roughness is much smaller than the pores, $l_{w} \ll l$. This means that the fields in the sub-domain $\Omega_{w}$ vary on the length scale $l_{w}$, whereas the fields in the bulk domain $\Omega_{s}$ vary on a much larger length scale, $l$. The wavelength, $\lambda$, of the roughnesses is much smaller than the radius of curvature of the smoothed interface $1 / \kappa$, so that the closure problem can be solved once for a flat surface and mapped onto the curved surface.

- The subdomain $\Omega_{w}$ can be represented locally by a periodic structure made of cells denoted $\Omega_{w_{i}}$, as shown in Fig. 2.

The first step of the method consists in building an approximate solution of the flow close to the rough wall, in the subdomain $\Omega_{w}$. Following ideas of mapping used in porous media sciences and homogenization techniques for the estimation of spatial or temporal deviations from averaged quantities, $\left(p_{w}, \mathbf{u}_{w}\right)$ is mapped linearly to the tangential components of the shear stress vector on $\Gamma_{w s}$ as

$p_{w}(\mathbf{x})=\mu_{\gamma} \mathbf{a}(\mathbf{x}) \cdot\left[\left.\mathbf{n} \cdot\left(\nabla \mathbf{u}_{s}+\left(\nabla \mathbf{u}_{s}\right)^{T}\right) \cdot(\mathbf{I}-\mathbf{n n})\right|_{\Gamma_{w s}}\right]$ in $\Omega_{w_{i}}$,

$\mathbf{u}_{w}(\mathbf{x})=\mathbf{A}(\mathbf{x}) \cdot\left[\left.\mathbf{n} \cdot\left(\nabla \mathbf{u}_{s}+\left(\nabla \mathbf{u}_{s}\right)^{T}\right) \cdot(\mathbf{I}-\mathbf{n n})\right|_{\Gamma_{\mathrm{ws}}}\right] \quad$ in $\quad \Omega_{w_{i}}$, 
where (a,A) are solutions of a mathematical problem that is solved over the representative cell $\Omega_{w_{i}}$. The normal vector $\mathbf{n}$ on $\Gamma_{w s}$ is uniform and oriented from the domain $\Omega_{w}$ to the domain $\Omega_{s}$. In these equations, we consider that the couple $\left(p_{w}, \mathbf{u}_{w}\right)$ is mapped to the tangential components, $\mathbf{n} \cdot\left(\nabla \mathbf{u}_{s}+\left(\nabla \mathbf{u}_{s}\right)^{T}\right) \cdot(\mathbf{I}-\mathbf{n n})$ of the shear stress vector $\mathbf{n} \cdot\left(\nabla \mathbf{u}_{s}+\left(\nabla \mathbf{u}_{s}\right)^{T}\right)$ on $\Gamma_{\text {ws }}$, assuming that the normal component of the velocity can be neglected under the conditions stated above. We also consider that variations of $\mathbf{n} \cdot\left(\nabla \mathbf{u}_{s}+\left(\nabla \mathbf{u}_{s}\right)^{T}\right) \cdot(\mathbf{I}-\mathbf{n n})$ on $\Gamma_{\text {ws }}$ occur on a lengthscale that is much larger than the wavelength of the roughness, $\lambda$. This is because the creeping flow in $\Omega_{w}$ implies that variations induced by the roughnesses rapidly attenuate away from the wall (see Kamrin et al., 2010 for analytical solution showing an exponential decrease). The shear stress vector is therefore treated as approximately constant over each unit-cell and the point on $\Gamma_{w_{i}}$ at which it is evaluated for the mappings Eqs. (2) and (3) is not fundamentally important. As an example, we could map the velocities and pressures at point $\mathbf{x}$ to the shear stress vector evaluated at point $\mathbf{x}^{\prime}$, defined so that $\mathbf{x}-\mathbf{x}^{\prime}$ and $\mathbf{n}$ are collinear. We could also use the surface average of the stress vector evaluated at the nearest point on $\Gamma_{\text {ws }}$. In Eqs. (2) and (3) and in the remainder of this work, we use the generic notation $\left.\mathbf{n} \cdot\left(\nabla \mathbf{u}_{s}+\left(\nabla \mathbf{u}_{s}\right)^{T}\right) \cdot(\mathbf{I}-\mathbf{n n})\right|_{\Gamma_{w s}}$ with the idea that locally, in each unit-cell, the stress vector can be treated as constant so that the mapping can be done to any point in $\Omega_{w}$ and any point on $\Gamma_{w s}$.

The corresponding local problem reads

$\nabla \cdot \mathbf{A}=0 \quad$ in $\quad \Omega_{w_{i}}$

$0=-\nabla \mathbf{a}+\nabla^{2} \mathbf{A}$ in $\Omega_{w_{i}}$,

$\mathbf{A}=0$ on $\Gamma_{\gamma \sigma}$,

$-\mathbf{a n}+\mathbf{n} \cdot\left(\nabla \mathbf{A}+(\nabla \mathbf{A})^{T}\right)=\mathbf{I}-\mathbf{n n}$ on $\Gamma_{w s}$,

$\mathbf{a}(\mathbf{r})=\mathbf{a}\left(\mathbf{r}+\lambda \mathbf{e}_{i}\right) \quad i=1, m-1$,

$\mathbf{A}(\mathbf{r})=\mathbf{A}\left(\mathbf{r}+\lambda \mathbf{e}_{i}\right) \quad i=1, m-1$,

where $\lambda$ is the wavelength and $\mathbf{e}_{i}$ are the two orthonormal unit vectors for directions tangential to the surface $\Gamma_{\text {ws }}$. Here, the transpose of the third-order tensor $(\nabla \mathbf{A})^{T}$ is defined as $\left[(\nabla \mathbf{A})^{T}\right]_{i j k}=\partial_{j} A_{i k}$ with $i, j, k \in \llbracket 1, m \rrbracket(m=2,3$ in our calculations). Recalling the mapping of $\mathbf{u}_{w}$, Eq. (3), we can write at any point on $\Gamma_{w s}$

$\mathbf{u}_{w}=\left.\mathbf{A}\right|_{\Gamma_{w s}} \cdot\left[\left.\mathbf{n} \cdot\left(\nabla \mathbf{u}_{s}+\left(\nabla \mathbf{u}_{s}\right)^{T}\right) \cdot(\mathbf{I}-\mathbf{n n})\right|_{\Gamma_{w s}}\right]$.

As discussed before, for $\Gamma_{\text {ws }}$ placed sufficiently far away from the rough wall, $\left.\mathbf{A}\right|_{\Gamma_{\mathrm{ws}}}$ is approximately constant in the tangential directions and may be computed as the surface average of the field $\mathbf{A}$ over the interface $\Gamma_{\text {ws }}$ of the periodic unit-cell.

Using the continuity of the velocities, $\mathbf{u}_{s}=\mathbf{u}_{w}$ on the interface $\Gamma_{w s}$, we then have the form of a generalized Navier condition, where $\mathbf{M}=\left.\mathbf{A}\right|_{\Gamma_{w s}}$ is a second-order tensor that can be interpreted in terms of slip coefficients along the directions tangential to the interface,

$\mathbf{u}_{w}=\mathbf{M} \cdot\left[\left.\mathbf{n} \cdot\left(\nabla \mathbf{u}_{s}+\left(\nabla \mathbf{u}_{s}\right)^{T}\right) \cdot(\mathbf{I}-\mathbf{n n})\right|_{\Gamma_{w s}}\right] \quad$ on $\quad \Gamma_{w s}$.

This parameter is also referred to as the mobility tensor in the work of Kamrin et al. (2010).

The flow problem in $\Omega_{s}$ finally reads $\nabla \cdot \mathbf{u}_{s}=0$ in $\Omega_{s}$,

$\rho_{s}\left(\frac{\partial \mathbf{u}_{s}}{\partial t}+\mathbf{u}_{s} \cdot \nabla \mathbf{u}_{s}\right)=-\nabla p_{s}+\nabla \cdot\left[\mu_{\gamma}\left(\nabla \mathbf{u}_{s}+\left(\nabla \mathbf{u}_{s}\right)^{T}\right)\right]+\rho_{\gamma} \mathbf{g}$

$\mathbf{u}_{s}=\mathbf{M} \cdot\left[\mathbf{n} \cdot\left(\nabla \mathbf{u}_{s}+\left(\nabla \mathbf{u}_{s}\right)^{T}\right) \cdot(\mathbf{I}-\mathbf{n n})\right] \quad$ on $\quad \Gamma_{\text {ws }}$.

Fig. 3 shows the different domains and the change in boundary condition. We remark that the accuracy of the linear relation Eq. (7c) between the velocity in the vicinity of the wall and the shear stress vector on $\Gamma_{\text {ws }}$ will strongly depend on the type of flow. The solution is accurate for a Couette flow, but only roughly approximates the quadratic profile of a Poiseuille flow within $\Omega_{w}$. In general, this implies that the error increases with the distance to the rough surface and that we must have $l_{w} \ll l$ in order to minimize the part of the flow that is linearly approximated. This aspect is further detailed in the computations Section 4.2.

Finally, the position of $\Gamma_{\text {ws }}$ can also be modified a posteriori, as first discussed by Veran et al. (2009). Consistent with the firstorder closure in Eqs. (4), we write the first-order Taylor series expansion

$\mathbf{u}_{s}\left(\mathbf{x}_{2}\right)=\mathbf{u}_{s}\left(\mathbf{x}_{1}\right)+\left.\left(\mathbf{x}_{\mathbf{2}}-\mathbf{x}_{\mathbf{1}}\right) \cdot\left(\nabla \mathbf{u}_{s}+\left(\nabla \mathbf{u}_{s}\right)^{T}\right)\right|_{\mathbf{x}_{1}}+\mathcal{O}\left(\left\|\mathbf{x}_{\mathbf{2}}-\mathbf{x}_{\mathbf{1}}\right\|^{2}\right)$,

with $\mathbf{x}_{1}-\mathbf{x}_{2}$ the displacement of the interface and $\mathbf{x}_{1}$ the reference position corresponding to Eq. (7c). If we move the interface in the normal direction, $\mathbf{x}_{\mathbf{1}}-\mathbf{x}_{\mathbf{2}}=\mathbf{n} \Delta x$, and if we consider that $\mathbf{u}_{s}$ remains tangential, we can write

$\mathbf{u}_{s}\left(\mathbf{x}_{2}\right) \simeq \mathbf{u}_{s}\left(\mathbf{x}_{1}\right)-(\Delta x \mathbf{I}) \cdot\left[\left.\mathbf{n} \cdot\left(\nabla \mathbf{u}_{s}+\left(\nabla \mathbf{u}_{s}\right)^{T}\right)\right|_{\mathbf{x}_{1}} \cdot(\mathbf{I}-\mathbf{n n})\right]$

Injecting $\mathbf{u}_{s}\left(\mathbf{x}_{1}\right)$ from Eq. (7c) into Eq. (9) yields

$\mathbf{u}_{s}\left(\mathbf{x}_{2}\right) \simeq(\mathbf{M}-\Delta x \mathbf{I}) \cdot\left[\mathbf{n} \cdot\left(\nabla \mathbf{u}_{s}+\left(\nabla \mathbf{u}_{s}\right)^{T}\right) \cdot(\mathbf{I}-\mathbf{n n})\right]$.

This step, which is described in detail in Veran et al. (2009) or Introini et al. (2011), can impact the accuracy of the Navier-slip boundary condition (see also Veran et al., 2009 or Introini et al., 2011), as we will demonstrate for a specific example later on. It is particularly useful in two-dimensional cases or isotropic configurations in order to derive an equivalent effective no-slip condition, which is obtained by choosing $\Delta x$ so that $M-\Delta x=0$ in Eq. (10) ( $\mathbf{M}$ is a scalar in 2D). This specific position of the effective boundary is interesting, mostly for its simplicity of implementation in a computational framework. However, we emphasize that such a position does not exist in three-dimensional cases if the roughness is anisotropic. If this is the case, the slip lengths are different in all directions and a tensorial form of the Navier-condition is necessary.
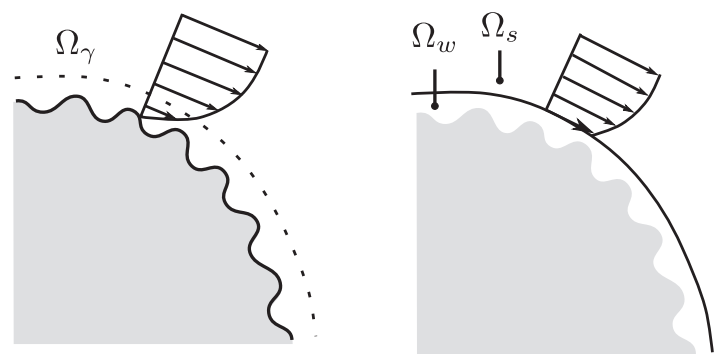

Fig. 3. Schematics of the rough domain $\Omega_{\gamma}$, on the left-hand side, and the effective domain $\Omega_{s}$, on the right-hand side. 


\section{Upscaling at the Darcy-scale via volume averaging}

Now that we have derived an effective smooth condition that replaces the rough wall, we can further upscale the system of equations, Eqs. (7), to obtain a homogenized system at the Darcy-scale. We use the method of volume averaging that has been utilized before to study this problem in the viscous and inertial regimes. In this approach, the partial differential equations at the microscale are averaged in space and approximate solutions for the velocity and pressure perturbations are determined using scaling arguments. When applied to Stokes equations, this process yields Darcy's law with a permeability tensor that can be calculated from the numerical resolution of a closure problem. Quasi-linearized Navier-Stokes equations can be treated in a similar manner, leading to an additional drag and a generalized Darcy-Forchheimer law (Whitaker, 1996) (generalized in the sense that anisotropy is included and that inertial terms are not necessarily quadratic). The development closely follows the early work of Whitaker (1986) and the more recent work of Lasseux et al. (2014) for the homogenization of a low-pressure gas phase in porous media with a similar slip condition on the walls, although tensorial in our case.

\subsection{Notations}

For a tensor $\psi_{i}$, where $i$ is the phase, we define the superficial average of $\psi_{i}$ at point $\mathbf{x}$ as

$\left\langle\psi_{i}\right\rangle(\mathbf{x}, t)=\frac{1}{|\mathcal{V}|} \int_{\mathcal{V}_{i}(\mathbf{x})} \psi_{i}(\mathbf{r}, t) d \mathbf{r}$,

where $\mathcal{V}_{i}(\mathbf{x})$ ( $i$ can be $s, \gamma$ or $w$ for each corresponding domain) is the averaging volume centered at $\mathbf{x}$ and $\mathcal{V}_{i}(\mathbf{x})=\mathcal{V}(\mathbf{x}) \cap \Omega_{i}$. The intrinsic average is defined as

$\left\langle\psi_{i}\right\rangle^{i}=\frac{1}{\left|\mathcal{V}_{i}\right|} \int_{\mathcal{V}_{i}} \psi_{i} d \mathbf{r}$,

so that the two definitions are related by

$\left\langle\psi_{i}\right\rangle=\phi_{i}\left\langle\psi_{i}\right\rangle^{i}$,

where the volume fraction of the domain $\Omega_{i}, \phi_{i}$, is defined as

$\phi_{i}=\frac{\left|\mathcal{V}_{i}\right|}{|\mathcal{V}|}$

We use the perturbation decomposition (see Whitaker, 1969; Gray and Majid Hassanizadeh, 1998)

$\psi_{i}=\left\langle\psi_{i}\right\rangle^{i}+\tilde{\psi}_{i}$,

where the deviation $\tilde{\psi}_{i}$ varies on a length scale $l$ and the intrinsic average $\left\langle\psi_{i}\right\rangle^{i}$ varies on a length scale $L$, such that $l \ll r_{0} \ll L$. We further require that (see e.g. Whitaker, 1999)

$\left\langle\tilde{\psi}_{i}\right\rangle \simeq 0$.

\subsection{Temporal averaging}

For sufficiently high Reynolds numbers, when the flow becomes fully unsteady, a macroscopic formulation of momentum transport generally requires both spatial and temporal averaging. In theory, the order in which these operators are applied is unimportant. The process should lead to the exact same results, whether the equations are first averaged in space or time. In practice, however, the combination of approximations that are used during upscaling often depends on the averaging order (see de Lemos, 2006; Nakayama and Kuwahara, 2008 for further details). Here, we start by averaging the pore-scale equations in time.
When unsteady flow develops, we use a time averaging operator for the field $\phi(\mathbf{x}, t)$ defined as

$\bar{\phi}(\mathbf{x}, t)=\frac{1}{\Delta T} \int_{t-\Delta T / 2}^{t+\Delta T / 2} \phi(\mathbf{x}, \tau) d \tau$,

where $\Delta T$ is the integration interval. We also use a standard average plus perturbation decomposition as

$\phi=\bar{\phi}+\phi^{\prime}$.

With these, we can average Eqs. (7) in time and obtain Reynolds stresses in the equations, a detailed analysis of this operation is given in de Lemos (2006). In this study, we primarily concentrate on regimes for which the time fluctuations are of low amplitude. Therefore, we neglect terms involving the product $\mathbf{u}^{\prime} \mathbf{u}^{\prime}$. In the fully turbulent regime, further theoretical developments should account for transient effects at the macro-scale. One way to deal with this issue could be an approach similar to that in Soulaine and Quintard (2014), where the system is first averaged in time using a standard closure for the Reynolds stress to model the turbulence at the micro-scale, before averaging the model in space.

With our approximations, the time-averaged mathematical problem is then

$\nabla \cdot \overline{\mathbf{u}}_{s}=0$ in $\Omega_{s}$,

$\rho_{\gamma}\left(\frac{\partial \overline{\mathbf{u}}_{s}}{\partial t}+\overline{\mathbf{u}}_{s} \cdot \nabla \overline{\mathbf{u}}_{s}\right)=-\nabla \bar{p}_{s}+\mu_{\gamma} \nabla^{2} \overline{\mathbf{u}}_{s}+\rho_{\gamma} \mathbf{g}$ in $\Omega_{s}$,

$\overline{\mathbf{u}}_{s}=\mathbf{M} \cdot\left[\mathbf{n} \cdot\left(\nabla \overline{\mathbf{u}}_{s}+\left(\nabla \overline{\mathbf{u}}_{s}\right)^{T}\right) \cdot(\mathbf{I}-\mathbf{n n})\right] \quad$ on $\quad \Gamma_{w s}$.

For simplicity, we remove the overline notation in the remainder of the paper, with the pressure and velocity fields implicitly understood as averaged in time.

\subsection{Spatial averaging}

In this section, the system of equations Eqs. (19) is upscaled using the method of volume averaging. Since the method is similar to the original work of Whitaker (1986) leading to Darcy's law (see also Quintard and Whitaker, 1994c,b,a), the details of the developments are in Appendix A. The Darcy-scale mass and momentum balance equations read

$\nabla \cdot\left\langle\mathbf{u}_{s}\right\rangle=0$

$\left\langle\mathbf{u}_{s}\right\rangle=-\frac{\mathbf{K}_{s}^{\star}}{\mu_{\gamma}} \cdot\left(\nabla\left\langle p_{s}\right\rangle^{s}-\rho_{\gamma} \mathbf{g}\right)$,

where $\mathbf{K}_{s}^{\star}$ is the apparent permeability (Edwards et al., 1990), which is computed from the resolution of a closure problem. This equation is obtained using an approximate form of the pressure and velocity perturbations, $\tilde{p}_{s}$ and $\tilde{\mathbf{u}}_{s}$, which are mapped linearly to $\left\langle\mathbf{u}_{s}\right\rangle^{s}$ as

$\tilde{\mathbf{u}}_{s}=\mathbf{B}_{s} \cdot\left\langle\mathbf{u}_{s}\right\rangle^{s}$,

$\tilde{p}_{s}=\mu_{\gamma} \mathbf{b}_{s} \cdot\left\langle\mathbf{u}_{s}\right\rangle^{s}$,

where $\left(\mathbf{b}_{s}, \mathbf{B}_{s}\right)$ are closure/mapping variables. $\mathbf{K}_{s}^{\star}$ can be computed from $\mathbf{b}_{s}$ and $\mathbf{B}_{s}$ as

$\mathbf{K}_{s}^{\star-1} \phi_{s}=-\frac{1}{\left|\mathcal{V}_{s}\right|} \int_{\mathcal{V} \cap \Gamma_{w s}} \mathbf{n} \cdot\left[-\mathbf{b}_{s} \mathbf{I}+\left(\nabla \mathbf{B}_{s}+\left(\nabla \mathbf{B}_{s}\right)^{T}\right)\right] d \mathbf{r}$.

For a viscous incompressible flow, it is well established that the flow at the macro-scale is described by Darcy's law. The presence of inertial effects at the micro-scale, as is the case in this study, yields an additional drag and a deviation from the classical Darcy's law. A correction, usually termed the Forchheimer correction 
(Whitaker, 1996), can be used to approximate the additional drag. The form of this correction has been widely studied in the literature, both numerically and experimentally. It is now known that different regimes can be distinguished for increasing Reynolds numbers (Firdaouss et al., 1997; Lage and Antohe, 2000; Skjetne and Auriault, 1999), even though the transition and the form of the corrections most likely depend on the porous structure (Lasseux et al., 2011). To recover a generalized form of the Darcy-Forchheimer equation, we decompose the tensor $\mathbf{K}_{s}^{\star}$ as

$\mathbf{K}_{s}^{\star}=\mathbf{K}_{s} \cdot\left(\mathbf{I}+\mathbf{F}_{s}\right)^{-1}$,

where $\mathbf{K}_{s}$ is the intrinsic permeability of the effective domain and the second part, $\mathbf{F}_{s}$, is the inertial correction. We then obtain

$\left\langle\mathbf{u}_{s}\right\rangle=-\frac{\mathbf{K}_{s}}{\mu_{\gamma}} \cdot\left(\nabla\left\langle p_{s}\right\rangle^{s}-\rho_{\gamma} \mathbf{g}\right)-\mathbf{F}_{s} \cdot\left\langle\mathbf{u}_{s}\right\rangle$.

Here, we emphasize that the permeability $\mathbf{K}_{s}^{\star}$ corresponds to the permeability of the effective smooth domain, $\Omega_{s}$, not to the initial domain, $\Omega_{\gamma}$. To recover the exact velocity field in $\Omega_{\gamma}$ and the corresponding permeability, consider the following expression of the velocity field,

$\mathbf{u}_{\gamma}=\chi_{w} \mathbf{u}_{w}+\chi_{s} \mathbf{u}_{s}$

where $\chi_{w}$ and $\chi_{s}$ are phase indicator functions of the domains $\Omega_{w}$ and $\Omega_{s}$ respectively, so that we have

$\left\langle\mathbf{u}_{\gamma}\right\rangle=\phi_{w}\left\langle\mathbf{u}_{w}\right\rangle^{w}+\phi_{s}\left\langle\mathbf{u}_{s}\right\rangle^{s}$.

We can simplify this expression using the fact that the domain $\Omega_{w}$ is a relatively small subdomain close to the no-slip wall, $\Gamma_{\gamma \sigma}$. Therefore, we have $\phi_{w} \ll \phi_{s}$ and consider that $\left\langle\mathbf{u}_{w}\right\rangle^{w} \ll\left\langle\mathbf{u}_{s}\right\rangle^{s}$, so that

$\left\langle\mathbf{u}_{\gamma}\right\rangle \simeq \phi_{s}\left\langle\mathbf{u}_{s}\right\rangle^{s}$

For the pressure, we assume that there is a small perturbation of the pressure within $\Omega_{s}$ so that

$\left\langle p_{\gamma}\right\rangle^{\gamma} \simeq\left\langle p_{s}\right\rangle^{s}$

With these, we can write

$\left\langle\mathbf{u}_{\gamma}\right\rangle=-\frac{\mathbf{K}_{s}}{\mu_{\gamma}} \cdot\left(\nabla\left\langle p_{\gamma}\right\rangle^{\gamma}-\rho_{\gamma} \mathbf{g}\right)-\mathbf{F}_{s} \cdot\left\langle\mathbf{u}_{\gamma}\right\rangle$.

The impact of these approximations is illustrated and discussed in the results of the next section.

\section{Validation and application to structured packings}

In this section, we compare our methodology to direct numerical simulations in various geometries of rough porous media. Our primary objective is to answer the following questions:

- Is our method efficient for a wide range of Reynolds number?

- How does the apparent permeability of the porous medium depend on the Reynolds number?

- What is the impact of different classes of roughness on the permeability?

In order to validate the method, we first focus on a creeping flow in a two-dimensional channel with rough walls. We discuss the impact of the roughness and of the position of the slip condition on the results, a study that is reminiscent of the analysis by Veran et al. (2009). We then go on to investigate the case of an inertial flow in a 2D array of cylinders with sinusoidal roughness, assessing the impact of the roughness on the permeability for Stokes and inertial flows. The methodology is finally applied to an element of structured packing with different classes of roughness and a tensorial slip.

\subsection{Methods}

\subsubsection{Boundary conditions}

Here, we describe the different boundary conditions used for applications. We have the rough, $\Omega_{\gamma}$, and effective, $\Omega_{s}$, domains. We also introduce the smooth domain $\Omega_{\text {smooth }}$, which is similar to $\Omega_{\gamma}$, but with smooth walls $\Gamma_{\gamma \sigma}^{\text {smooth }}$ positioned at the averaged position of the roughnesses. Fig. 4 shows these three configurations.

A no-slip condition applies to the boundaries $\Gamma_{\gamma \sigma}$ and $\Gamma_{\gamma \sigma}^{\text {smooth }}$

$\boldsymbol{u}_{\gamma}=0$,

while the effective condition Eq. (6) applies at the smooth wall $\Gamma_{w s}$

$\mathbf{u}_{s}=\mathbf{M} \cdot\left[\mathbf{n} \cdot\left(\nabla \mathbf{u}_{s}+\left(\nabla \mathbf{u}_{s}\right)^{T}\right) \cdot(\mathbf{I}-\mathbf{n n})\right] \quad$ on $\Gamma_{w s}$.

Recall that $\Gamma_{w s}$ can be placed arbitrarily using the Taylor series approximation with the conditions detailed in Section 2.2.

\subsubsection{General algorithm}

We summarize here the methodology used for the different geometries considered in this section. Our goal is to compare results obtained with the upscaling methods with those of direct numerical simulations. The different steps are as follows:

1. Direct numerical simulations in the domains $\Omega_{\gamma}$ and $\Omega_{\text {smooth }}$.

2. Upscaling approach:

(a) Definition of a representative element for the roughness, $\Omega_{w_{i}}$.

(b) Determination of the effective condition via the resolution of a local boundary value problem over $\Omega_{w_{i}}$.

(c) Computation of the velocity field $\mathbf{u}_{s}$ in $\Omega_{s}$.

(d) Resolution of the closure problem for the determination of $\mathbf{K}_{s}^{\star}$.

3. Comparison of the permeabilities $\mathbf{K}_{\gamma}^{\star}$ and $\mathbf{K}_{\text {smooth }}^{\star}$ computed from point 1 to $\mathbf{K}_{s}^{\star}$ computed from point 2 .

\subsubsection{Numerical schemes}

Two different methods are applied for the resolution of the closure problems. For the creeping flow in the two-dimensional rough channel, the boundary value problem Eqs. (4) and the closure problem Eqs. (65) (in Appendix A) are solved using standard finite element methods. Stokes equations are discretized using Lagrange quadratic elements for the velocity and linear elements for the pressure (P2/P1). A stabilization term is also added in the equations to fix the constant part of the pressure. We use the generic solver FreeFem++ (Hecht, 2013), which provides a flexible environment where the grid and the rough geometry can be easily modified.

More demanding simulations in the inertial regimes, for both the two-dimensional array of cylinders and the threedimensional structured packings, are performed using the standard finite volume methods in OpenFoam ${ }^{\circledR}$. The closure problem Eqs. (65) was implemented using the solver simpleFoam (SIMPLE algorithm, Patankar, 1980) with the tensorial Navier condition derived from the slip boundary conditions (named slip in OpenFoam ${ }^{\circledR}$, see Ferrás et al., 2013 for more detail).

As required for the resolution of the closure problem, the velocity field is determined using direct numerical simulations of the flow via the solver icoFoam (PISO algorithm, Issa, 1986), with a volume source term and periodic boundary conditions. All calculations were performed using the HPC resources of CALMIP supercomputing center with 160 cores for the most demanding 

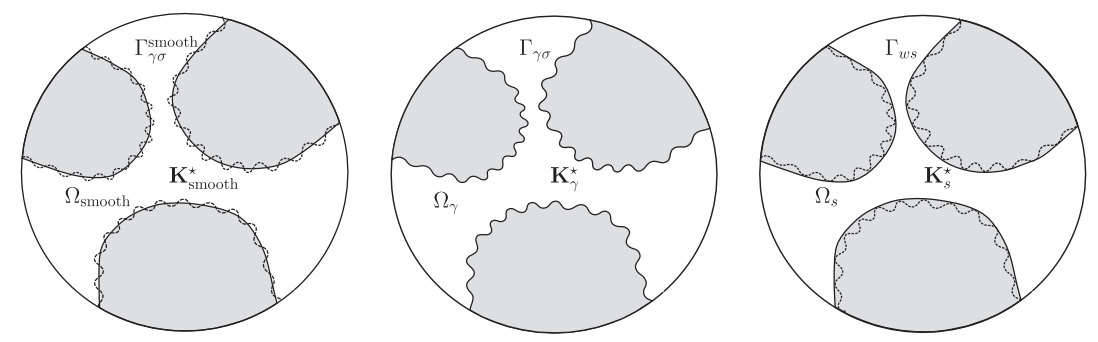

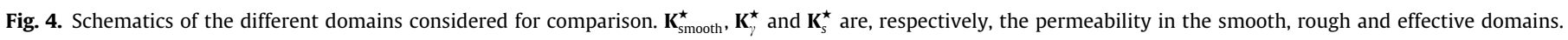

simulations. The CPU time (number of cores $\times$ computing time) required up to $10^{5} \mathrm{~h}$ for all calculations.

\subsubsection{Assessing the accuracy of the three-dimensional direct numerical simulations}

Three-dimensional direct numerical simulations are carried out from the creeping flow until the onset of unsteady flow. To estimate the accuracy of our solutions on a fixed grid, we use an approach similar to that described by Jin et al. (2015), who introduced the following quantity $\Delta$,

$\Delta=\frac{g_{p}-g_{s}}{g_{p}}$,

where $g_{p}$ and $g_{s}$ are two ways of defining the pressure gradient. $g_{p}$ corresponds to the global pressure gradient across the simulated geometry, whereas $g_{s}$ is determined by integrating the local dissipation rate over the fluid domain,

$g_{s}=\frac{\mu_{\gamma}}{2\left|\mathcal{V}_{s}\right|\left\|\left\langle\mathbf{u}_{s}\right\rangle^{s}\right\|} \int_{\mathcal{V}_{s}} \nabla \mathbf{u}_{s}: \nabla \mathbf{u}_{s} d \mathbf{r}$.

Note that the same definition also holds for the domains $\Omega_{\gamma}$ and $\Omega_{\text {smooth }}$, and the corresponding averaging volumes $\mathcal{V}_{\gamma}$ and $\mathcal{V}_{\text {smooth }}$.

In theory, $\Delta=0$. However, if the grid size does not resolve the smallest scales of the flow, the global dissipation rate is undervalued and $\Delta>0$. Therefore, the accuracy of the calculations can be evaluated by calculating $\Delta$ and, following studies in Jin et al. (2015), we consider that the calculations are accurate when $\Delta$ is below 10\% (see Jin et al., 2015 for further detail).

\subsubsection{Definition of the REV and boundary conditions}

A precise definition of the REV and whether or not it should consist of one or several periodic unit-cells for turbulent flows is a delicate issue. For instance, the idea that the porous medium filters the longest length scales of the turbulence has been widely used for simulations in structured packings (Raynal and RoyonLebeaud, 2007; Ahmadi et al., 2012; Soulaine and Quintard, 2014). Few results of large scale simulations are available in the literature (see Owens et al., 2013; Nikou and Ehsani, 2008) and most of the simulations are limited to steady state. For arrays of squares, Jin et al. (2015) have recently shown that the porous structure does indeed impose a cut-off in the characteristic length scales of the flow. They further study the spatial correlations of the velocity field while changing the number of unit-cells included in the REV, showing that the largest possible structures of the flow are about the size of the pores, even for large values of the porosity. This suggests that calculations over a single unit-cell with periodic boundary conditions recover most of the flow features for a broad range of porosities. This question of the size of the REV is addressed further by the results presented in Agnaou et al. (2016) about the flow stability and Hopf bifurcation in arrays of cylinders. Their results indicate that the transition to unsteady flow is sensitive to the number of unit cells included in the REV for porosities larger than $\sim 50 \%$ ( $>55 \%$ in Fig. 10 in Agnaou et al. (2016), arrays of squares). These two studies seem to indicate that:

1. for values of the porosity less than $50 \%$, a single unit-cell with periodic conditions captures both the stability and the structure of the flow,

2. for larger values of the porosity, a single unit-cell captures most of the features of the flow but may fail to accurately describe its stability and the transition to unsteady flow.

In this section, we will study a variety of porous media including ones with porosities larger than $50 \%$. In all cases, we chose to limit our computations to a single unit-cell with periodic boundary conditions to minimize the computational cost. This is because our primary objective is not to study stability and transitions, but the ability of our models to recover the spatial structure of the flow.

\subsubsection{Definition of the Reynolds number}

To analyze the effect of inertial flow, we define here a set of dimensionless Reynolds numbers. The difficulty in doing so is mostly the choice of the characteristic length scale. In isotropic porous media, a common choice is the square root, $\sqrt{K}$, of the intrinsic permeability, $K$, so that

$\operatorname{Re}_{k}=\frac{\rho_{\gamma}\left\|\left\langle\mathbf{u}_{\gamma}\right\rangle\right\| \sqrt{K}}{\mu_{\gamma}}$.

This length scale has several advantages over the average pore-size $l$. First, when the variance of the pore-size distribution is large and the porous medium consists of large voids and small pore-throats, defining a unique length scale that characterizes the flow at large Reynolds number is difficult. $\sqrt{K}$ is an evaluation of such a unique length scale, but one that characterizes the flow at small Reynolds number and is directly connected to distribution of viscous dissipation inside the porous structure (Zami-Pierre et al., 2016). Second, it has been shown that this definition of the Reynolds number captures more accurately the transition to the inertial regime (Lasseux et al., 2011; Andrade et al., 1999). For comparison with the literature in chemical engineering, we also define the following Reynolds number

$\operatorname{Re}_{h}=\frac{\rho_{\gamma}\left\|\left\langle\mathbf{u}_{\gamma}\right\rangle\right\| h}{\mu_{\gamma}}$,

where $h$ is a geometrical length scale. We will use $h=d$ with $d$ the diameter of the cylinder in the case of the rough cylinder and $h=H$ for the structured packings.

\subsection{Application to a channel with sinusoidal walls}

The methodology is first applied to a creeping flow within a two-dimensional channel with sinusoidal walls. In a twodimensional configuration, the effective boundary condition, Eq. (6), reads 
$u_{s} \mathbf{e}_{x}=M_{x x} \frac{\partial u_{s}}{\partial y} \mathbf{e}_{x}$

where $M_{x x}$ is the Navier coefficient (or equivalently the slip length). In order to assess the effect of the distance of the effective surface to the rough wall on the accuracy of the method, we apply the methodology for multiple positions (A-G in Fig. 5). The effective condition corresponding to each position is derived from Eq. (38) using the Taylor series Eq. (10). Position B corresponds to the effective no-slip position, i.e. the displacement of the interface that leads to $M_{x x}-\Delta x=0$. The permeability $K_{s}$, normalized to the exact permeability $K_{\gamma}$ of the rough channel, is given in Fig. 5 along with the different positions considered. It is shown that displacing the effective boundary closer to the rough wall improves the accuracy, and that the effective no-slip condition (line B) is the most accurate configuration, as previously discussed in Veran et al. (2009).

We then fix the position of the effective boundary at the no-slip position and vary the amplitude and wavelength of the sinusoid. The effective and exact permeabilities, normalized to the intrinsic permeability of a Poiseuille flow, $K=\frac{H^{2}}{12}$, are presented in Fig. 6 . We observe several limit cases. First, when the amplitude tends to zero, we recover the permeability of the flat case. Second, when the wavelength tends to zero, we recover the permeability of an equivalent flat surface positioned on top of the sinusoid. Third, when the wavelength is much larger than the height of the channel $H$, two different situations are observed. If both sinusoids are in phase (blue curve in Fig. 6), we have $k_{\gamma} \rightarrow 1$, whereas if there is a phase shift of $\frac{\pi}{2}$ ( red $^{1}$ curve in Fig. 6) another limit is attained, $k_{\gamma \text {-shift }}<1$. This is because, for a fixed value of the amplitude, this geometry generates a bottleneck effect with flow focusing and defocusing even in the limit of large wavelengths. The effective condition accurately captures the large variations in the permeability (about $25-50 \%$ ) induced by the roughness, except in the specific case of the asymmetry between both boundaries. In this case, there is a strong coupling between the pore flow and the boundaries and the approximations needed for the derivation of the slip boundary condition, in particular the constraint $l_{w} / l \ll 1$, are not valid.

\subsection{Application to an array of rough cylinders}

We now apply the effective boundary approach to a twodimensional array of rough cylinders, as shown in Fig. 7, both in the Stokes and inertial regimes. In order to minimize the error induced by the effective surface theory, the boundary $\Gamma_{w s}$ is placed at the no-slip position. Example 2D meshes used for the effective and rough domains are given in Appendix B.

\subsubsection{Flow regimes}

Results are presented in Figs. 8 and 9 for a cylinder of diameter $d=0.6 H\left(\phi_{\gamma} \approx 0.72\right)$, where $H$ is the unit-cell width. As discussed previously in Lasseux et al. (2011), the transition between the Darcean and the fully turbulent regime is complex and strongly depends on the geometry of the porous structure. Here, we identify five different regimes as follows.

- For $R e_{k} \lesssim 0.3\left(R e_{d} \lesssim 1.7\right)$, the flow is Darcean and the apparent permeability is constant (velocity field in Fig. 10a).

- For $0.3<R e_{k}<2\left(1.7<R e_{d}<11.5\right)$, we observe a weakly inertial regime where $F$ scales in $R e_{k}^{2}$. This regime has been widely referenced in the literature (Firdaouss et al., 1997; Lage and Antohe, 2000).

\footnotetext{
${ }^{1}$ For interpretation of color in 'Fig. 6', the reader is referred to the web version of
} this article.

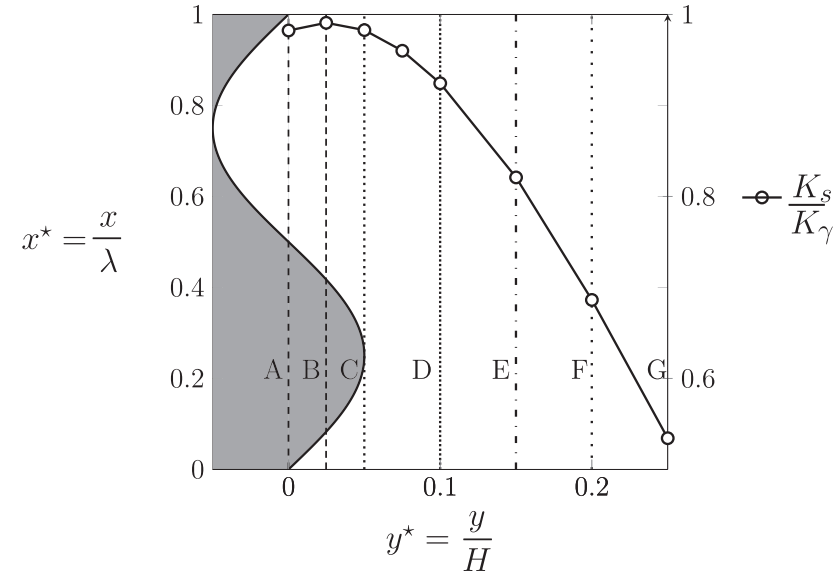

Fig. 5. Permeability $K_{s}$ of the effective domain non-dimensionalized with the permeability $K_{\gamma}$ from the exact geometry, as a function of the distance $y$ from the effective surface to the sinusoïdal wall, normalized with $H$, the height of the channel. The effective surface is positioned from $A\left(y^{\star}=0\right)$ to $G\left(y^{\star}=0.25\right)$. Accurate results are obtained for the equivalent "no-slip" position (line B). Line A corresponds to the mean position of the sinusoid, $\Gamma_{\gamma \sigma}^{\text {smooth }}$.

- For $3<R e_{k}<20\left(17<R e_{d}<114\right)$, a strongly inertial regime develops (velocity field in Fig. 10c), which is characterized by a slowdown in the growth of $F$, see (Lasseux et al., 2011).

- For $R e_{k} \simeq 25\left(R e_{d} \simeq 172\right)$, there is a steep increase in the correction $F$ that reflects the transition from steady to unsteady flow. A plot of the volume averaged velocity $\left\langle\mathbf{u}_{s}\right\rangle$ without timeaveraging is given in Fig. 12 and velocity fields at different times are presented in Fig. 11, highlighting the cyclic behavior of two large vortices. This transition is described in detail by Koch and Ladd (1997) for a similar two-dimensional geometry where they show that the pressure drag exhibits a sudden increase.

- For large Reynolds numbers, $R e_{k}>50\left(R e_{d}>285\right)$, the flow is completely unsteady (velocity field in Figs. 10c and f). For the few points that we computed, we observe that $F$ scales linearly with $R e_{k}$ and the pressure drag, $-\int_{\Gamma_{\gamma \sigma}} \mathbf{n} \cdot\left(p_{\gamma} \mathbf{I}\right) d \mathbf{r}$, dominates over the viscous drag. To further understand the unsteady regime and the impact of temporal fluctuations on the results, additional computations for larger values of the Reynolds number are needed. Such simulations should also be performed in threedimensional configurations since two-dimensional computations usually fail to recover important features of turbulent flows.

Independently from the slip condition, we emphasize several important results from these direct numerical simulations. First, although we observe a decrease in the slope of $F$ in the strongly inertial regime $\left(3<R e_{k}<20\right)$, we did not recover a scaling where the correction $F$ is linear with $\operatorname{Re}_{k}$. This was already observed for simple arrays of cylinders in Lasseux et al. (2011), and they also found that the introduction of disorder favors the emergence of a quadratic regime. Second, we observe that the standard Forchheimer correction, where the drag scales with $\operatorname{Re}_{k}^{2}$ ( $F$ is linear with $R e_{k}$ ), seems to be valid for the onset of the unsteady regime when $R e_{k}$ is just above 50 . This may suggest that the Darcy-Forchheimer formulation actually corresponds to a time-averaged macro-scale equation, although further analysis and simulations at larger Reynolds numbers are necessary. This slow transition from the Darcy to the Forchheimer regime is in contrast with some previous works in the literature (Firdaouss et al., 1997; Lage and Antohe, 2000) where the transition regime is neglected and the drag rapidly becomes quadratic. These effects have been considered negligible for practical applications in previous works (see Lage, 1998; Bejan and Nield, 2013). However, our results on this simple unit cell show that the drag is not quadratic for a significant range of 

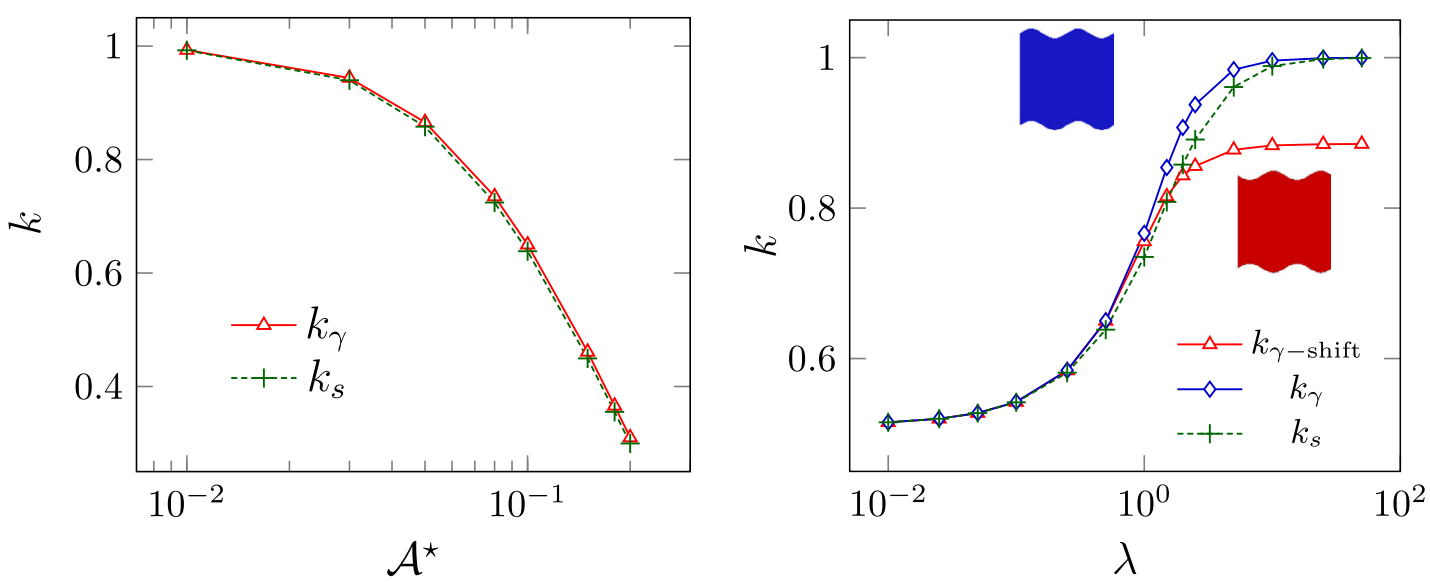

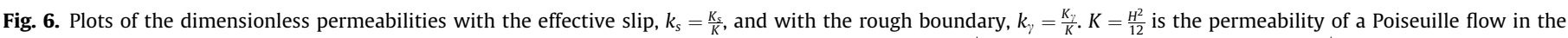

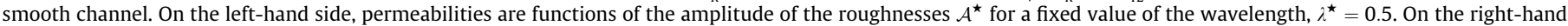
side, permeabilities are functions of the wavelength $\lambda^{\star}$ for a fixed value of the amplitude, $\mathcal{A}^{\star}=0.1$.

Reynolds numbers (about a decade). As previously mentioned, the introduction of disorder in arrays of squares favors the emergence of a quadratic regime (Lasseux et al., 2011), which may explain why Forchheimer corrections can be used with good accuracy for disordered media over a broad range of Reynolds number (see discussion in Lage et al. (1997) and Clavier et al. (2015)). This emphasizes the importance of the pore space topology on these results, which should be revisited for more complex and disordered three-dimensional structures.

\subsubsection{Impact of the slip condition}

For the slip condition, we see that the effective approach accurately captures the impact of the roughness on the permeability for $R e_{k} \lesssim 40$. For larger Reynolds number, however, the roughness generates additional inertial effects and viscous dissipation that are not captured by the effective condition; a difference that is expected to be further amplified for larger Reynolds numbers for various reasons. First, the effective boundary condition, as presented in this paper, uses a creeping flow approximation within the boundary layer and is therefore limited to moderate Reynolds numbers. In addition, unless the roughness is so small as to be included in the viscous sublayer, the flow perturbations induced by the wall in the turbulent regime will propagate to the center of the pores, a phenomenon lying outside the domain of validity of the model. Finally, the closure problem is quasi-stationary, which is only true for $R e_{k}<25$. Beyond this point, the velocity field $\mathbf{u}_{s}$ is time-averaged for the resolution of the closure problem and further theoretical developments are required to accurately cap-

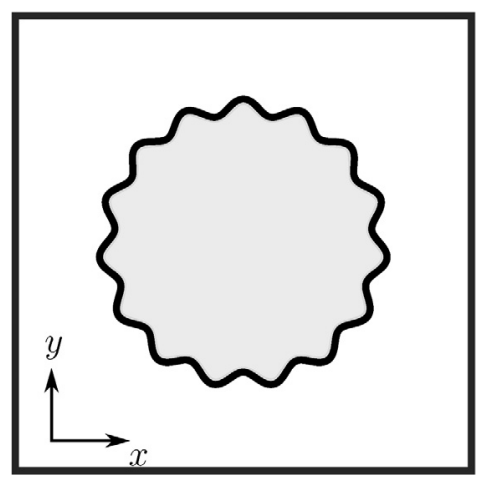

Fig. 7. Illustration of a unit-cell for a 2D array of rough cylinders.

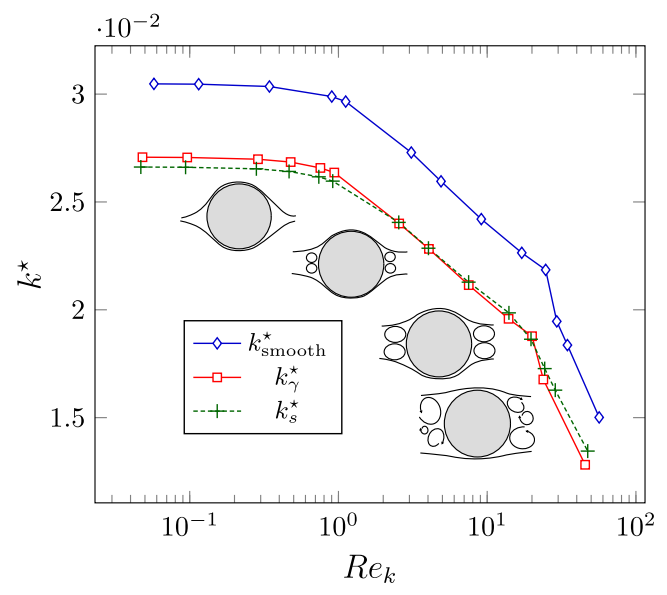

Fig. 8. Dimensionless apparent permeability $k^{\star}$ along the $x$-axis as a function of the Reynolds number for the effective condition $k_{s}^{\star}=\frac{K_{s}^{\star}}{d^{2}}$, the rough $k_{\gamma}^{\star}=\frac{K_{\gamma}^{\star}}{d^{2}}$ and smooth $k_{\text {smooth }}^{\star}=\frac{K_{\text {smooth }}^{\star}}{d^{2}}$ cylinders.

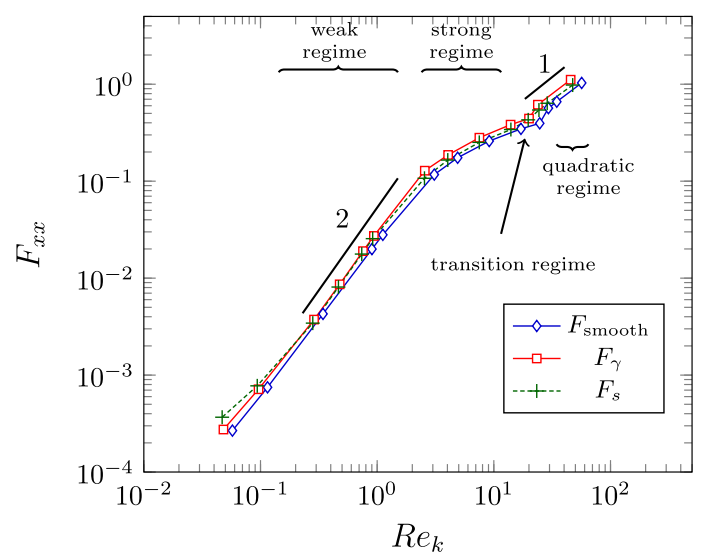

Fig. 9. Correction coefficient $F_{x x}$ as a function of the Reynolds number for the effective, rough, and smooth domains.

ture the effects of fluctuations in time. For instance, macro-scale equations involving time convolutions have been used previously to capture high-frequency temporal fluctuations of the average fields (see discussion in Davit and Quintard (2012) regarding dispersion effects). 


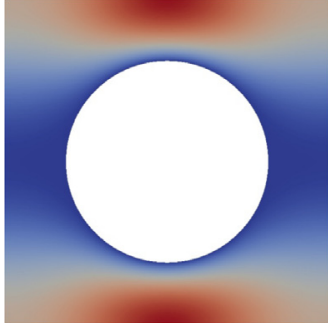

(a) Stokes regime

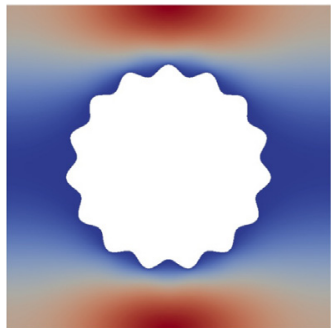

(d) Stokes regime

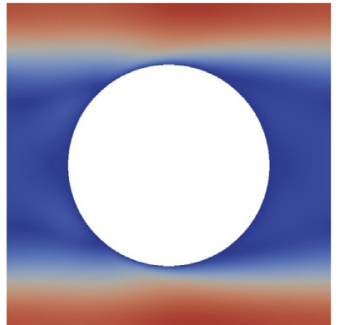

(b) $R e_{k}=14$

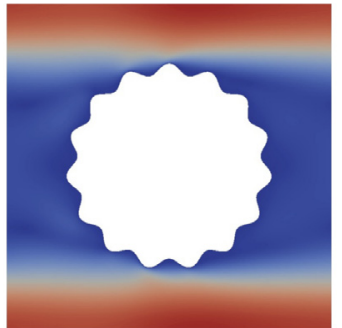

(e) $R e_{k}=14$

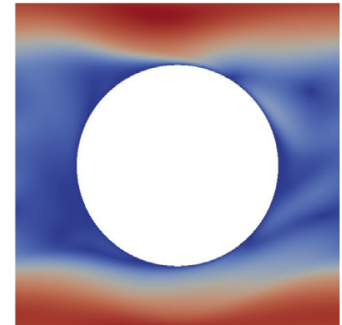

(c) $R e_{k}=56$

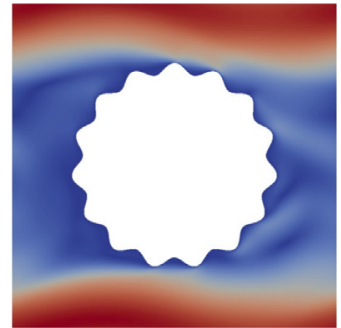

(f) $R e_{k}=56$

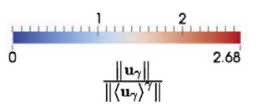

Fig. 10. Norm of the velocity fields in the domains $\Omega_{s}$ (top) and $\Omega_{\gamma}$ (bottom).

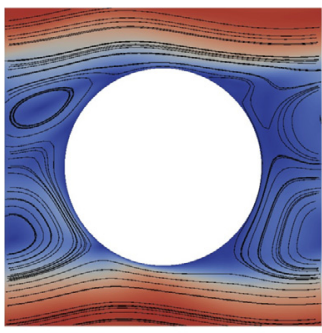

(a)

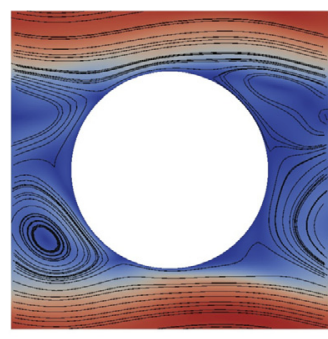

(b)

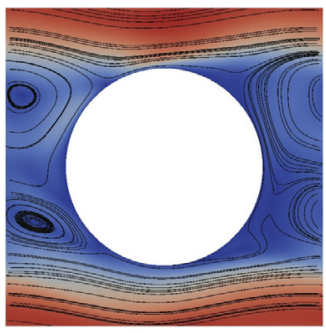

(c)

Fig. 11. Velocity streamlines in the domains $\Omega_{s}$ for $R e_{k}=56$ at three different times. The oscillation motion of the vortices is seen from left to right.

To evaluate the effect of the ratio $l_{w} / l$, we further study the impact of the porosity, and therefore the size of the pores, on the accuracy of the slip boundary condition for a viscous flow. Results are plotted in Fig. 13. Consistent with the case of the sinusoidal

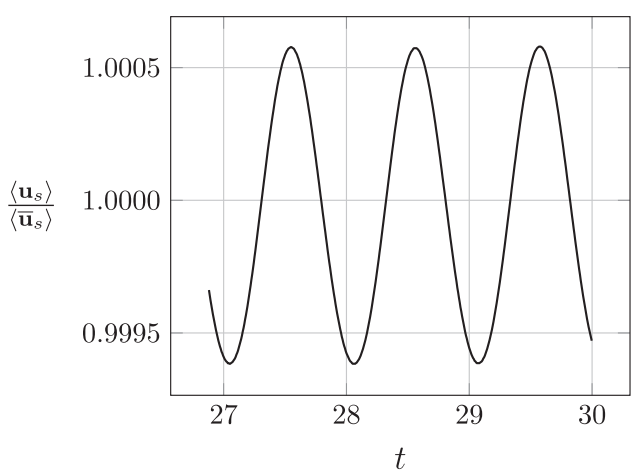

Fig. 12. Volume averaged velocity $\left\langle\mathbf{u}_{s}\right\rangle$ as a function of time for $\operatorname{Re}_{k}=29$ $\left(R e_{d}=168\right)$. wall, we see that the accuracy of the slip boundary condition decreases as the porosity decreases and $l_{w} / l$ increases.

\subsection{Application to structured packings}

We now consider a geometry representative of structured packings used in separation processes. The flow of the gas phase in such packings is the focus of a number of studies in the literature (see Raynal and Royon-Lebeaud, 2007; Ahmadi et al., 2012; Soulaine and Quintard, 2014; Owens et al., 2013), using primarily computational fluid dynamics. Recently, Soulaine and Quintard (2014) proposed a detailed analysis of the upscaling procedure for momentum transport and derived a generalized DarcyForchheimer law at the macro-scale for the gas phase that can be used, theoretically, for relatively high Reynolds numbers. They showed that the permeability tensor, $\mathbf{K}^{\star}$, has a null row and column describing the absence of flow in the direction orthogonal to the corrugated sheets. They also found that the amplitude of the non-diagonal terms is negligible compared to diagonal ones and that the diagonal terms on $\mathbf{K}^{\star}$ are identical. This was also 


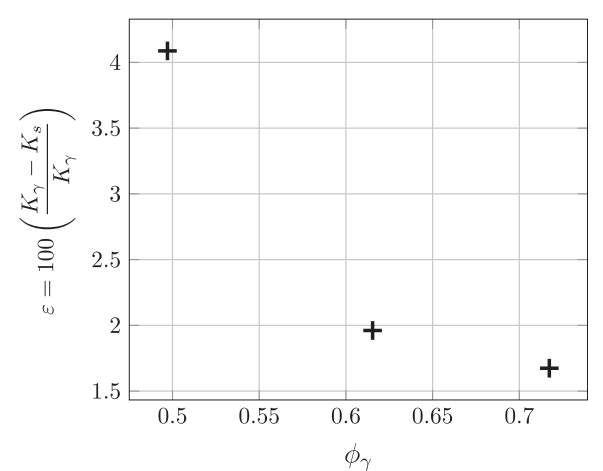

Fig. 13. Error between exact and effective intrinsic permeabilities for three porosity levels. Cylinder diameters from left to right: $d / H=0.8,\left(l_{w} / l=0.0250\right) d / H=0.7$, $\left(l_{w} / l=0.0167\right)$, and $d / H=0.6\left(l_{w} / l=0.0125\right)$.

assumed in earlier works by Mahr and Mewes (2007) and Raynal and Royon-Lebeaud (2007). The tensor $\mathbf{K}^{\star}$ is therefore written as

$$
\mathbf{K}^{\star}=K^{\star}\left(\begin{array}{lll}
1 & 0 & 0 \\
0 & 1 & 0 \\
0 & 0 & 0
\end{array}\right),
$$

with the $z$-axis normal to the corrugated sheets. This form of the tensor $\mathbf{K}^{\star}$, however, is only valid for specific symmetries of the local structure. The surface roughness, either generated by a traveling train of waves at the liquid-gas interface (Alekseenko et al., 2009; Vellingiri et al., 2013) or engineered as a textured corrugated sheet, may break symmetries of the unit-cell. We therefore consider a more general form of the tensor as

$$
\mathbf{K}^{\star}=\left(\begin{array}{ccc}
K_{x x}^{\star} & K_{y x}^{\star} & 0 \\
K_{x y}^{\star} & K_{y y}^{\star} & 0 \\
0 & 0 & 0
\end{array}\right) .
$$

In this section, we explore the impact of three different orientations and profiles of rough patterns on the coefficients of $\mathbf{K}^{\star}$ (see Fig. 14).

The geometries are now three-dimensional, so that we need the full slip tensor $\mathbf{M}$ of the Navier condition

$\mathbf{u}_{s}=\mathbf{M} \cdot\left[\mathbf{n} \cdot\left(\nabla \mathbf{u}_{s}+\left(\nabla \mathbf{u}_{s}\right)^{T}\right) \cdot(\mathbf{I}-\mathbf{n n})\right]$.

In order to limit the error introduced by the linear approximation, the effective surface is positioned on the top of the roughness for all the cases treated in this section. The geometry $a$ describes a wall texture that can be found in structured packings (McGlamery, 1988) to control the liquid phase dispersion. The rough pattern $b$ is identical to the profile $a$, but rotated $45^{\circ}$ to study the impact of an inclined pattern on the isotropy of the permeability tensor. The geometry $c$ represents a train of soliton waves propagating on the surface of a thin liquid film along the axis of the column.

\subsubsection{Flow regimes}

Direct numerical computations are performed in all cases (see Fig. 15) with the quality of the numerics evaluated as described in Section 4.1 .4 (see Table 1 for the unit-cell $a$ ) and the mesh of the geometry $a$ in Appendix B. Results are presented in Fig. 16 for the permeability and Fig. 17 for the Forchheimer term. Similarly to the rough cylinder, different regimes can be identified depending on the Reynolds number. We recover weak and strong inertial regimes, with a scaling of $F$ in $R e_{k}^{2}$ in the weak regime $\left(0.8<R e_{k}<4\right)$. The transition to unsteady flow occurs for $\operatorname{Re}_{k} \sim 40$ leading to a significant loss in permeability, both in the smooth and rough domains. Consistent with the twodimensional observations for the rough cylinders, the effective

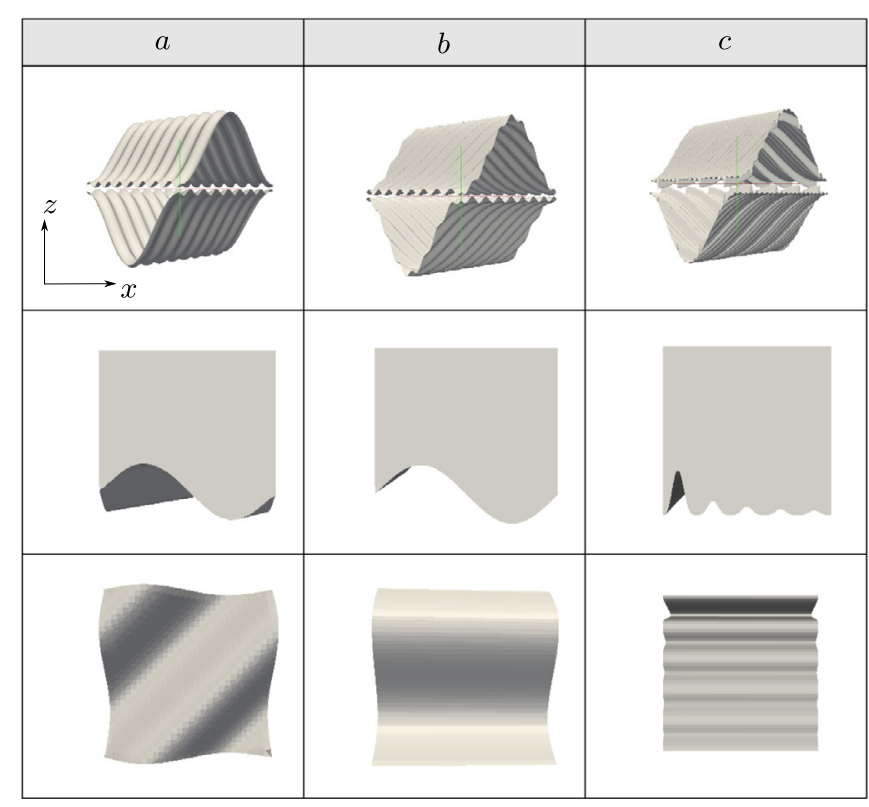

Fig. 14. Geometries considered in the simulations. The first line shows the unitcells of structured packings, while the second and third lines are the rough elements $\Omega_{w_{i}}$ on the surface. Roughness types $a$ and $b$ correspond to typical wall-textures that can be engineered on the surface. Roughness $c$ represents a traveling train of soliton waves along the column axis.

domain method accurately captures the pressure drop, until the transition for $\operatorname{Re}_{k} \sim 40\left(\operatorname{Re}_{H} \sim 500\right)$. As discussed previously, this regime cannot be captured by the effective boundary condition developed in this paper. This is particularly true for the geometry $c$, which generates an important reduction of permeability, even in the Stokes regime, and yields strong inertial effects. These effects are shown in Fig. 17, where we observe a correction in the effective domain that is similar to the smooth case, further suggesting that the slip boundary does not recover the perturbations of the flow generated at the small scale by the rough wall and propagating to the center of the pores.

\subsubsection{Impact of the slip condition}

We now compare the exact permeabilities for the smooth and rough geometries, $\mathbf{K}_{\text {smooth }}^{\star}$ and $\mathbf{K}_{\gamma}^{\star}$, to the permeability resulting from the up-scaling analysis $\mathbf{K}_{s}^{\star}$. Results of the simulations in a large range of Reynolds numbers are presented in Fig. 16 for the permeability coefficients $K_{s_{a}}^{\star}$ and $K_{s_{c}}^{\star}$ along the axis of the column, $y$, non-dimensionalized with the characteristic length $H=0.01$. For the geometry $a$, the slip coefficients of the effective tensor $\mathbf{M}$ are identical along the $x$ and $y$ axis, $M_{x x}=M_{y y}=7.9 \times 10^{-5} \mathrm{~m}$. The consequence of this symmetry in the slip tensor is a symmetry in the intrinsic permeability tensor $\mathbf{K}_{\mathbf{s}_{\mathbf{a}}}$, with $K_{s_{a}}^{x x}=K_{s_{a}}^{y y}$. The coefficients $K_{s_{a}}^{x x}=K_{s_{a}}^{y y} \simeq 7.1 \times 10^{-7} \mathrm{~m}^{2}$ are of the same order of magnitude as those in Soulaine and Quintard (2014). In agreement with (Soulaine and Quintard, 2014), the non-diagonal terms are negligible compared to diagonal ones, which indicates that this specific structure of packings does not generate anisotropic effects. For the geometry $b$, we have $M_{x x}>M_{y y}$, with $M_{x x}=1.2 \times 10^{-4} \mathrm{~m}$ and $M_{y y}=7.5 \times 10^{-5} \mathrm{~m}$, leading to different permeability coefficients along $x$ and $y$, with $K_{s_{b}}^{x x} \simeq 7.1 \times 10^{-7} \mathrm{~m}^{2}$ and $K_{s_{b}}^{y y}=6.9 \times 10^{-7} \mathrm{~m}^{2}$. For the geometry $c$, the tensor $\mathbf{M}$ also exhibits different diagonal coefficients, $M_{x x}=8.1 \times 10^{-5} \mathrm{~m}$ and $M_{y y}=1.8 \times 10^{-5}$, which lead to $K_{s_{c}}^{x x} \simeq 7.1 \times 10^{-7} \mathrm{~m}^{2}$ and $K_{s_{c}}^{y y}=6.5 \times 10^{-7} \mathrm{~m}^{2}$ in the permeability tensor $\mathbf{K}_{\mathbf{s}_{\mathbf{c}}}$. For the geometry $b$, comparison between the exact and 


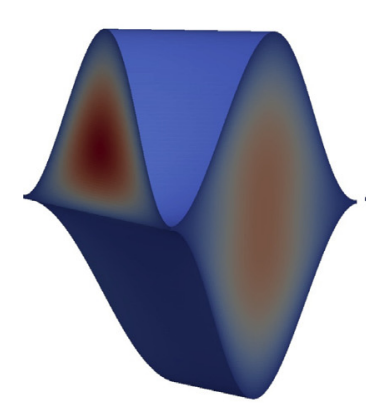

(a) Stokes regime

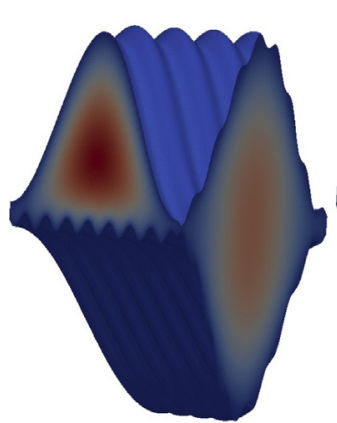

(d) Stokes regime

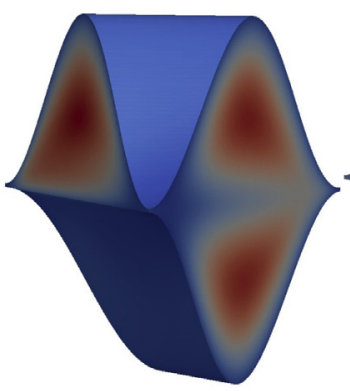

(b) $R e_{k}=7.5$

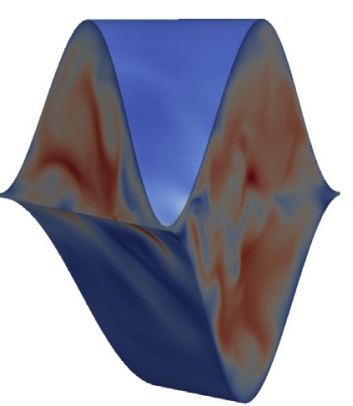

(c) $R e_{k}=85$

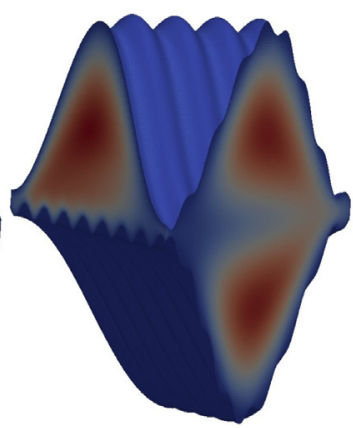

(e) $R e_{k}=7.5$

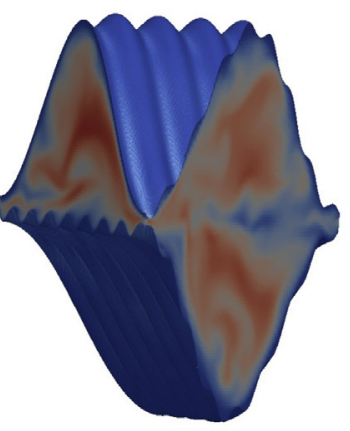

(f) $R e_{k}=85$

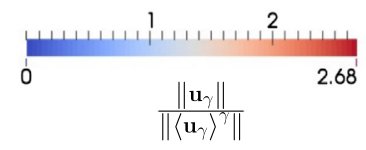

Fig. 15. Norm of the velocity fields in the domains $\Omega_{s}$ (top) and $\Omega_{\gamma}$ (bottom).

effective permeabilities are shown in Fig. 18 for the stationary regime. We find that the roughnesses $a$ and $b$ reduce the permeability of about $12 \%$, while the roughness $c$ induces a change of about $18 \%$. These results concur with the experimental data of Tsai (2010), who compared the dry pressure drop for a packing with and without roughness at the surface. For the geometry $b$, the effective domain method recovers the anisotropy with a lower permeability in the $x$-direction than in the $y$-direction in Fig. 18.

The primary reason for using the effective boundary condition, rather than the no-slip condition on the rough surface, was to make computations tractable. To evaluate the gain, we show a mesh convergence plot for simulations in the Stokes regime for the soliton case (geometry c) in Fig. 19. We see that we obtain a similar accuracy for both calculations only for a much finer mesh in the case of the rough surface. This is because the rough structure is composed of sharp soliton waves and we need a fine mesh to

Table 1

Numerical accuracy of the results for the unit-cell $a$.

\begin{tabular}{|c|c|c|c|c|c|}
\hline \multicolumn{3}{|c|}{ Mesh $\sim 120^{3}$ cells } & \multicolumn{3}{|c|}{ Accuracy } \\
\hline$\nabla\left\langle p_{\gamma}\right\rangle^{\gamma}$ & $R e_{k}$ & $R e_{H}$ & $g_{p}$ & $g_{s}$ & $\Delta$ \\
\hline 5 & 18 & 220 & 5 & 4.99 & 0.18 \\
\hline 10 & 34 & 402 & 10 & 9.93 & 0.62 \\
\hline 15 & 46 & 541 & 15 & 14.82 & 1.19 \\
\hline 20 & 48 & 574 & 20 & 19.71 & 1.47 \\
\hline 30 & 58 & 693 & 30 & 29.43 & 1.89 \\
\hline 50 & 79 & 940 & 50 & 47.37 & 5.22 \\
\hline 100 & 118 & 1401 & 100 & 91.28 & 8.72 \\
\hline
\end{tabular}

capture the flow over these structures. The consequences are twofold. First, obtaining a good quality mesh is much more difficult in the rough surface than in the smooth case. Second, the simulation times are longer for the rough case. For instance, the finest simulation (last point on Fig. 19) represents over $500 \mathrm{~h}$ of CPU time with the rough surface, whereas the equivalent simulation in the effective domain only represents about $120 \mathrm{~h}$ of CPU time.

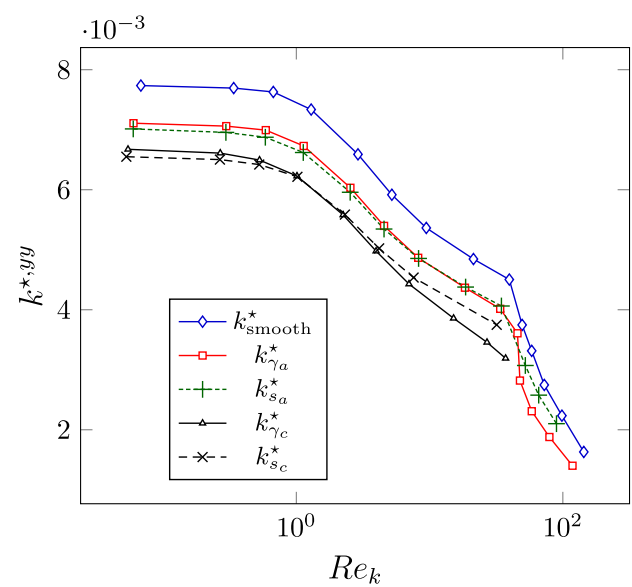

Fig. 16. Dimensionless permeability $k^{\star y y}$ along the $y$-axis for the geometries $a$ and $c$ as a function of the Reynolds number. The exact permeabilities of the smooth $k_{\text {smooth }}^{\star}=\frac{K_{s m o o t h}^{\star}}{H^{2}}$ and rough $k_{\gamma}^{\star}=\frac{K_{\gamma}^{\star}}{H^{2}}$ domains are compared to the permeability $k_{s}^{\star}=\frac{K_{s}^{\star}}{H^{2}}$ obtained with the tensorial slip condition. 


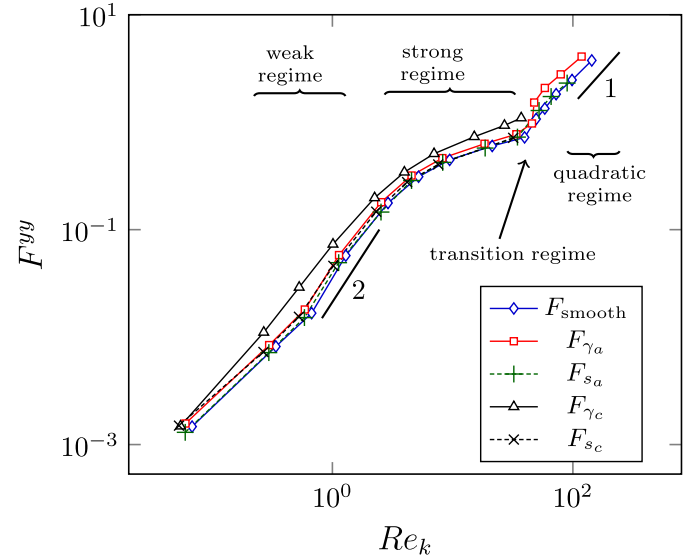

Fig. 17. Correction coefficient along the $y$-axis $F^{y y}$ for the geometries $a$ and $c$ as a function of the Reynolds number. The exact corrections of the smooth $F_{\text {smooth }}$ and rough $F_{\gamma}$ domains are compared to the correction $F_{s}$ obtained with the tensorial slip condition.

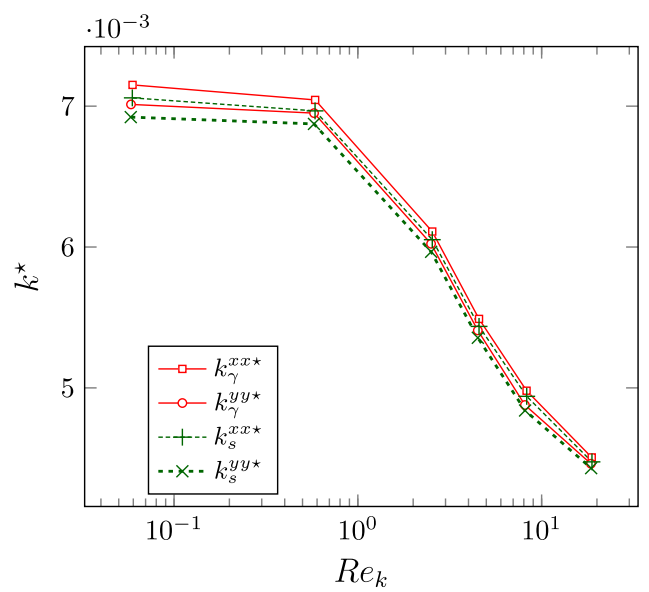

Fig. 18. Dimensionless permeability $k^{\star}$ coefficients in the $x$ and $y$ directions as functions of the Reynolds number for the geometry $b$. Results show good agreement between the exact and effective permeabilities with the anisotropy $k_{s}^{x \star \star}>k_{s}^{y y \star}$.

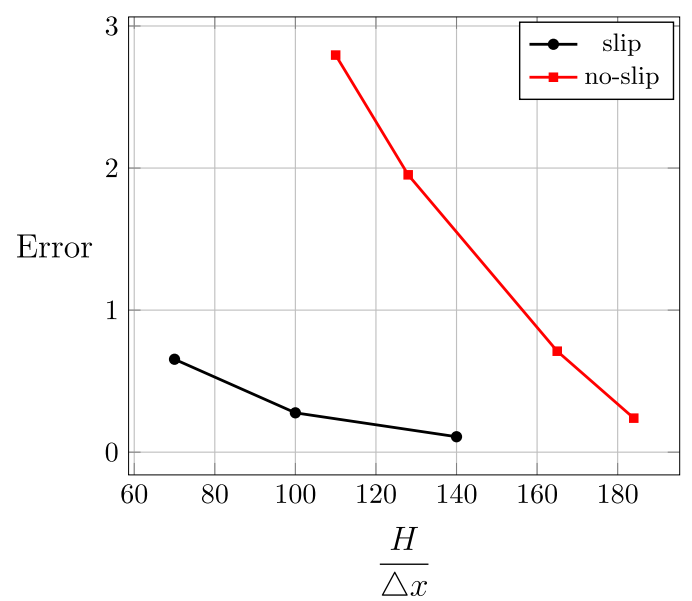

Fig. 19. Plot of the error as a function of the average number of mesh elements per direction, $\frac{H}{\Delta x}$. The error is defined $100\left(\left\|\frac{\left\langle u_{x}\right\rangle-\left\langle u_{x}\right\rangle_{R}}{\left\langle u_{x}\right\rangle_{R}}\right\|\right)$ where $\left.\left\langle u_{x}\right\rangle\right|_{R}$ is a reference average velocity obtained with an extremely fine mesh, $\frac{H}{\Delta x}=180$ for the smooth surface and $\frac{H}{\Delta x}=285$ for the rough surface

\section{Conclusion}

In this work, we provide a generic two-step methodology for the estimation of macro-scale properties in porous media with rough surfaces. The rough surface is first replaced by an effective smooth one with a slip boundary condition, which considerably simplifies computations in large domains. In three-dimensional configurations, the slip condition has a tensorial form that can capture surface anisotropy. Second, the micro-scale flow problem is upscaled at the Darcy-scale to obtain a generalized DarcyForchheimer formulation.

In applying our approach to a variety of geometries, we identified a number of fundamental aspects of flow in porous media with rough surfaces:

- even a small roughness can induce a significant decrease in permeability, suggesting that those complex surfaces, often treated as smooth ones, should be considered carefully in a multi-scale analysis.

- in the smooth, slip and rough cases, we have studied the evolution of the apparent permeability as a function of the Reynolds number. We show that several different regimes can be identified. These results suggest that the standard quadratic DarcyForchheimer formulation may be valid only for relatively large Reynolds numbers when the flow is fully unsteady. Computations of the flow for larger values of the Reynolds numbers are necessary to further understand this regime, along with the impact of temporal fluctuations on the permeability.

- the effective boundary condition accurately describes the permeability at the macro-scale for the Stokes and inertial flow regimes, but fails when unsteady flow develops. This is because, in this last regime, the impact of the roughness is not localized to the vicinity of the wall but propagates to the center of the pores. For large amplitudes of the roughness, our approach is therefore limited to relatively small Reynolds number. Further developments are needed to accurately model the flow characteristics at high Reynolds numbers.

\section{Acknowledgements}

This work was granted access to the HPC resources of CALMIP supercomputing center under the allocation 2015-P1547.

\section{Appendix A. Spatial averaging}

In this section, the system of equations Eqs. (19) is volume averaged using the definitions introduced in Section 3.1. We detail only the main steps of the developments, the reader is referred to the works of Lasseux et al. (2014) and Whitaker (1986) for further details or to other works (see e.g. Quintard and Whitaker, 1994c, b,a).

We use the following spatial averaging theorem (Howes and Whitaker, 1985) for the permutation between volume averaging and spatial differentiation

$\left\langle\nabla \psi_{i}\right\rangle=\nabla\left\langle\psi_{i}\right\rangle+\frac{1}{|\mathcal{V}|} \int_{\mathcal{V} \cap \Gamma} \mathbf{n} \psi_{i} d \mathbf{r}$

and similar for the divergence. The porosity of the medium is also considered as constant, so that

$\nabla \phi_{s}=-\frac{1}{|\mathcal{V}|} \int_{\mathcal{V} \cap \Gamma} \mathbf{n} d \mathbf{r}=0$.

The application of the volume averaging theorem, Eq. (42), to the continuity equation yields 
$\nabla \cdot\left\langle\mathbf{u}_{s}\right\rangle+\frac{1}{|\mathcal{V}|} \int_{\mathcal{V} \cap \Gamma_{w s}} \mathbf{n} \cdot \mathbf{u}_{s} d \mathbf{r}=0$.

The effective condition, Eq. (6), implies that the velocity on the boundary $\Gamma_{w s}$ is tangential, so that the integral term vanishes and the averaged continuity equations reads

$\nabla \cdot\left\langle\mathbf{u}_{s}\right\rangle=0$.

The momentum equation, Eq. (19b), may be written as

$\underbrace{\rho_{\gamma}\left[\frac{\partial \mathbf{u}_{s}}{\partial t}+\nabla \cdot\left(\mathbf{u}_{s} \mathbf{u}_{s}\right)\right]}_{\text {LHS }}=\underbrace{-\nabla p_{s}+\nabla \cdot\left[\mu_{\gamma}\left(\nabla \mathbf{u}_{s}+\left(\nabla \mathbf{u}_{s}\right)^{T}\right)\right]+\rho_{\gamma} \mathbf{g}}_{\text {RHS }}$ in $\Omega_{s}$,

which is decomposed, for clarity, into a left and a right hand side, LHS and RHS respectively. Averaging the LHS leads to

$\langle$ LHS $\rangle=\rho_{\gamma} \frac{\partial\left\langle\mathbf{u}_{s}\right\rangle}{\partial t}+\rho_{\gamma} \nabla \cdot\left\langle\mathbf{u}_{s} \mathbf{u}_{s}\right\rangle+\frac{1}{|\mathcal{V}|} \int_{\mathcal{V} \cap \Gamma_{\mathrm{ws}}} \mathbf{n} \cdot\left(\mathbf{u}_{s} \mathbf{u}_{s}\right) d \mathbf{r}$.

The slip condition on $\Gamma_{w s}$ implies that $\frac{1}{|\mathcal{V}|} \int_{\mathcal{V} \cap \Gamma_{w s}} \mathbf{n} \cdot\left(\mathbf{u}_{s} \mathbf{u}_{s}\right) d \mathbf{r}=0$. Using the relation between intrinsic and superficial averages Eq. (13) and the decomposition into average plus perturbation Eq. (15), this leads to

$\langle$ LHS $\rangle=\rho_{\gamma} \phi_{s} \frac{\partial\left\langle\mathbf{u}_{s}\right\rangle^{s}}{\partial t}+\rho_{\gamma} \nabla \cdot\left(\phi_{s}\left\langle\mathbf{u}_{s}\right\rangle^{s}\left\langle\mathbf{u}_{s}\right\rangle^{s}\right)+\rho_{\gamma} \nabla \cdot\left(\tilde{\mathbf{u}}_{s} \tilde{\mathbf{u}}_{s}\right)$,

or

$\langle\mathrm{LHS}\rangle=\rho_{\gamma} \phi_{s} \frac{\partial\left\langle\mathbf{u}_{s}\right\rangle^{s}}{\partial t}+\rho_{\gamma} \phi_{s}\left\langle\mathbf{u}_{s}\right\rangle^{s} \cdot \nabla\left\langle\mathbf{u}_{s}\right\rangle^{s}+\rho_{\gamma} \nabla \cdot\left(\tilde{\mathbf{u}}_{s} \tilde{\mathbf{u}}_{s}\right)$,

using the averaged mass conservation equation Eq. (45).

Averaging the RHS of Eq. (19b) yields

$$
\begin{aligned}
\langle\mathrm{RHS}\rangle= & -\nabla\left\langle p_{s}\right\rangle+\mu_{\gamma} \nabla^{2}\left\langle\mathbf{u}_{s}\right\rangle+\nabla \cdot\left(\frac{1}{|\mathcal{V}|} \int_{\mathcal{V} \cap \Gamma_{w s}} \mathbf{n u} \mathbf{u}_{s} d \mathbf{r}\right) \\
& +\frac{1}{|\mathcal{V}|} \int_{\mathcal{V} \cap \Gamma_{w s}} \mathbf{n} \cdot\left[-p_{s} \mathbf{I}+\mu_{\gamma}\left(\nabla \mathbf{u}_{s}+\left(\nabla \mathbf{u}_{s}\right)^{T}\right)\right] d \mathbf{r}+\rho_{\gamma} \mathbf{g} .
\end{aligned}
$$

Contrary to developments for a no-slip condition, the term $\nabla \cdot\left(\frac{1}{\mathcal{V} \mid} \int_{\mathcal{V} \cap \Gamma_{\mathrm{ws}}} \mathbf{n u}_{\mathrm{s}} d \mathbf{r}\right)$ cannot, strictly speaking, be eliminated (this issue was also studied in the work of Lasseux et al. (2014)). An order of magnitude estimation of this term compared to others in the equation leads to

$\nabla \cdot\left(\frac{1}{|\mathcal{V}|} \int_{\mathcal{V} \cap \Gamma_{\mathrm{ws}}} \mathbf{n} \mathbf{u}_{s} d \mathbf{r}\right) \sim O\left(\frac{\left\|\nabla \mathbf{u}_{s}\right\|}{L}\right) \ll\left(\frac{\left\|\nabla \mathbf{u}_{s}\right\|}{l}\right)$,

We therefore neglect this term.

Using the spatial decomposition Eq. (15), we have

$$
\begin{aligned}
\langle\mathrm{RHS}\rangle= & -\phi_{s} \nabla\left\langle p_{s}\right\rangle^{s}+\mu_{\gamma} \phi_{s} \nabla^{2}\left\langle\mathbf{u}_{s}\right\rangle^{s} \\
& +\frac{1}{|\mathcal{V}|} \int_{\mathcal{V} \cap \Gamma_{w s}} \mathbf{n} \cdot\left[-\tilde{p}_{s} \mathbf{I}+\mu_{\gamma}\left(\nabla \tilde{\mathbf{u}}_{s}+\left(\nabla \tilde{\mathbf{u}}_{s}\right)^{T}\right)\right] d \mathbf{r}+\rho_{\gamma} \mathbf{g} .
\end{aligned}
$$

Combining expressions for the LHS and RHS, we finally obtain

$$
\begin{aligned}
\rho_{\gamma} & \frac{\partial\left\langle\mathbf{u}_{s}\right\rangle^{s}}{\partial t}+\rho_{\gamma}\left\langle\mathbf{u}_{s}\right\rangle^{s} \cdot \nabla\left\langle\mathbf{u}_{s}\right\rangle^{s}+\rho_{\gamma} \phi_{s}^{-1} \nabla \cdot\left\langle\tilde{\mathbf{u}}_{s} \tilde{\mathbf{u}}_{s}\right\rangle \\
= & -\nabla\left\langle p_{s}\right\rangle^{s}+\mu_{\gamma} \nabla^{2}\left\langle\mathbf{u}_{s}\right\rangle^{s} \\
& +\frac{1}{\left|\mathcal{V}_{s}\right|} \int_{\mathcal{V} \cap \Gamma_{\text {ws }}} \mathbf{n} \cdot\left[-\tilde{p}_{s} \mathbf{I}+\mu_{\gamma}\left(\nabla \tilde{\mathbf{u}}_{s}+\left(\nabla \tilde{\mathbf{u}}_{s}\right)^{T}\right)\right] d \mathbf{r}+\rho_{\gamma} \mathbf{g} .
\end{aligned}
$$

As referred in Whitaker (1996), Soulaine and Quintard (2014), and Lasseux et al. (2014), the terms in the LHS of Eq. (53) as well as the diffusion term can be neglected compared to other terms, leading to $0=-\nabla\left\langle p_{s}\right\rangle^{s}+\rho_{\gamma} \mathbf{g}+\frac{1}{\left|\mathcal{V}_{s}\right|} \int_{\mathcal{V} \cap \Gamma_{\mathrm{ws}}} \mathbf{n} \cdot\left[-\tilde{p}_{s} \mathbf{I}+\mu_{\gamma}\left(\nabla \tilde{\mathbf{u}}_{s}+\left(\nabla \tilde{\mathbf{u}}_{s}\right)^{T}\right)\right] d \mathbf{r}$

\section{A.1. Mathematical problem governing the deviations}

The averaged form of the continuity equation Eq. (45) is subtracted from Eq. (19a) to obtain

$\nabla \cdot \tilde{\mathbf{u}}_{s}=0$.

Using the same approach, we can subtract the averaged momentum equation Eq. (53) from the pore-scale relation Eq. (19b). Along with the assumption of time-scale and length-scale separation, this yields

$$
\begin{aligned}
\rho_{\gamma}\left(\frac{\partial \tilde{\mathbf{u}}_{s}}{\partial t}+\mathbf{u}_{s} \cdot \nabla \tilde{\mathbf{u}}_{s}\right)= & -\nabla \tilde{p}_{s}+\mu_{\gamma} \nabla^{2} \tilde{\mathbf{u}}_{s} \\
& -\frac{1}{\left|\mathcal{V}_{s}\right|} \int_{\mathcal{V} \cap \Gamma_{w s}} \mathbf{n} \cdot\left[-\tilde{p}_{s} \mathbf{I}+\mu_{\gamma}\left(\nabla \tilde{\mathbf{u}}_{s}+\left(\nabla \tilde{\mathbf{u}}_{s}\right)^{T}\right)\right] d \mathbf{r} .
\end{aligned}
$$

Using the perturbation decomposition, the Navier condition Eq. (6) is also written

$\tilde{\mathbf{u}}_{s}+\mathbf{M} \cdot\left[\mathbf{n} \cdot\left(\nabla \tilde{\mathbf{u}}_{s}+\left(\nabla \tilde{\mathbf{u}}_{s}\right)^{T}\right) \cdot(\mathbf{I}-\mathbf{n n})\right]=-\left\langle\mathbf{u}_{s}\right\rangle^{s} \quad$ on $\quad \Gamma_{w s}$.

As indicated in Eq. (16), the average of the deviations over the fluid domain is zero, (Whitaker, 1986),

$\left\langle\tilde{p}_{s}\right\rangle^{s}=0 ; \quad\left\langle\tilde{\mathbf{u}}_{s}\right\rangle^{s}=0$

\section{A.2. Closure problem}

Identifying inhomogeneous source terms in the equations above, we can now formulate an approximate form for the perturbations. In this case, the only source term is the averaged velocity $\left\langle\mathbf{u}_{s}\right\rangle^{s}$ in Eq. (57), so that the perturbations can be mapped linearly to $\left\langle\mathbf{u}_{s}\right\rangle^{s}$ as

$\tilde{\mathbf{u}}_{s}=\mathbf{B}_{s} \cdot\left\langle\mathbf{u}_{s}\right\rangle^{s}$,

$\tilde{p}_{s}=\mu_{\gamma} \mathbf{b}_{s} \cdot\left\langle\mathbf{u}_{s}\right\rangle^{s}$

where the closure variables $\left(\mathbf{b}_{s}, \mathbf{B}_{s}\right)$ are first- and second-order tensors, respectively. By substituting Eqs. (59) and (60) into Eqs. (55)(57) and assuming that Eqs. (59) and (60) hold correct for any value of $\left\langle\mathbf{u}_{s}\right\rangle^{s}$, we obtain

$\nabla \cdot \mathbf{B}_{s}=0$ in $\Omega_{s}$,

$\rho_{\gamma} \mathbf{u}_{s} \cdot \nabla \mathbf{B}_{s}=-\nabla \mathbf{b}_{s}+\nabla^{2} \mathbf{B}_{s}$

$$
-\frac{1}{\left|\mathcal{V}_{s}\right|} \int_{\mathcal{V} \cap \Gamma_{\mathrm{ws}}} \mathbf{n} \cdot\left[-\mathbf{b}_{s} \mathbf{I}+\left(\nabla \mathbf{B}_{s}+\left(\nabla \mathbf{B}_{s}\right)^{T}\right)\right] d \mathbf{r},
$$

$\mathbf{B}_{s}+\mathbf{M} \cdot\left[\mathbf{n} \cdot\left(\nabla \mathbf{B}_{s}+\left(\nabla \mathbf{B}_{s}\right)^{T}\right) \cdot(\mathbf{I}-\mathbf{n n})\right]=-\mathbf{I}$ on $\quad \Gamma_{w s}$,

$\left\langle\mathbf{B}_{s}\right\rangle^{s}=0$,

$\mathbf{B}_{s}\left(\mathbf{r}+\mathbf{l}_{i}\right)=\mathbf{B}_{s}(\mathbf{r}) \quad i=1,2,3$,

$\mathbf{b}_{s}\left(\mathbf{r}+\mathbf{l}_{i}\right)=\mathbf{b}_{s}(\mathbf{r}) \quad i=1,2,3$,

where we have added the standard periodic condition for closure. This problem is integro-differential which can complicate numerical resolution. To simplify computations, we use the following change of variables (see also Whitaker, 1999 and Soulaine and Quintard, 2014) for $\mathbf{b}_{s}$ and $\mathbf{B}_{s}$, 
$\mathbf{B}_{s}^{0}=\left(\mathbf{B}_{s}+\mathbf{I}\right) \cdot \mathbf{K}_{s}^{\star} \phi_{s}^{-1}$,

$\mathbf{b}_{s}^{0}=\mathbf{b}_{s} \cdot \mathbf{K}_{s}^{\star} \phi_{s}^{-1}$

where $\mathbf{K}_{s}^{\star}$ is defined such as

$\mathbf{K}_{s}^{\star-1} \phi_{s}=-\frac{1}{\left|\mathcal{V}_{s}\right|} \int_{\mathcal{V} \cap \Gamma_{\mathrm{ws}}} \mathbf{n} \cdot\left[-\mathbf{b}_{s} \mathbf{I}+\left(\nabla \mathbf{B}_{s}+\left(\nabla \mathbf{B}_{s}\right)^{T}\right)\right] d \mathbf{r}$.

Injecting Eqs. (62) and (63) into the system of equations Eqs. (61) leads to

$\nabla \cdot \mathbf{B}_{s}^{0}=0$ in $\Omega_{s}$,

$\frac{\rho_{\gamma}}{\mu_{\gamma}} \mathbf{u}_{s} \cdot \nabla \mathbf{B}_{s}^{0}=-\nabla \mathbf{b}_{s}^{0}+\nabla^{2} \mathbf{B}_{s}^{0}+\mathbf{I}$ in $\Omega_{s}$,

$\mathbf{B}_{s}^{0}+\mathbf{M} \cdot \mathbf{n} \cdot\left(\nabla \mathbf{B}_{s}^{0}+\left(\nabla \mathbf{B}_{s}^{0}\right)^{T}\right) \cdot(\mathbf{I}-\mathbf{n n})=0$ on $\Gamma_{w s}$,

$\left\langle\mathbf{B}_{s}^{0}\right\rangle^{s}=\mathbf{K}_{s}^{\star} \phi_{s}^{-1}$

$\mathbf{B}_{s}^{0}\left(\mathbf{r}+\mathbf{l}_{i}\right)=\mathbf{B}_{s}^{0}(\mathbf{r}) \quad i=1,2$

$\mathbf{b}_{s}^{0}\left(\mathbf{r}+\mathbf{l}_{i}\right)=\mathbf{b}_{s}^{0}(\mathbf{r}) \quad i=1,2$.

This is a Navier-Stokes problem with the effective condition Eq. (65c) that can be solved using standard numerical methods. The components of the tensor $\mathbf{K}_{s}^{\star}$ are obtained by considering a source term along the different directions of space in the closure problem.
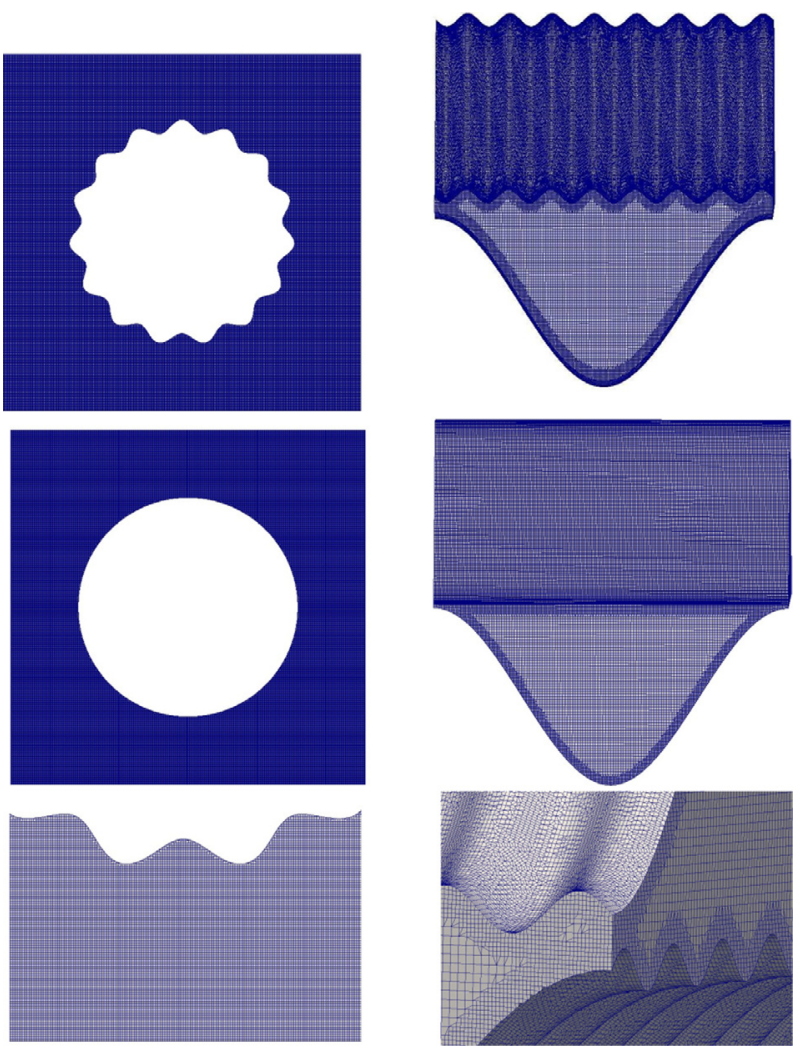

Fig. 20. Meshes for the unit-cell of the array of beads (left) and for the element of packings (right). Domains $\Omega_{\gamma}$ and $\Omega_{s}$ are given. The element of packings corresponds to the geometry $a$.
Similarly to the work in Soulaine and Quintard (2014), the problem still involves the micro-scale velocity field $\mathbf{u}_{s}$ that solves the set Eqs. (19) in the slip domain $\Omega_{s}$.

\section{Appendix B. Visualisation of the grids}

We show in Fig. 20 the grids of the exact and effective domains $\Omega_{\gamma}$ and $\Omega_{s}$, both for the 2D cylinder and the geometry $a$ of structured packings.

\section{References}

Achdou, Y., Pironneau, O., Valentin, F., 1998a. Effective boundary conditions for laminar flows over periodic rough boundaries. J. Comput. Phys. 147 (1), 187218

Achdou, Y., Le Tallec, P., Valentin, F., Pironneau, O., 1998b. Constructing wall laws with domain decomposition or asymptotic expansion techniques. Comput. Methods Appl. Mech. Eng. 151 (1-2), 215-232.

Agnaou, M., Lasseux, D., Ahmadi, A., 2016. From steady to unsteady laminar flow in model porous structures: an investigation of the first Hopf bifurcation. Comput. Fluids 136, 67-82.

Ahmadi, G., Hosseini, S.H., Shojaee, S., Zivdar, M., 2012. Computational fluid dynamics studies of dry and wet pressure drops in structured packings. J. Ind. Eng. Chem. 18 (4), 1465-1473.

Alekseenko, S.V., Aktershev, S.P. Cherdantsev, A.V., Kharlamov, S.M., Markovich, D. M., 2009. Primary instabilities of liquid film flow sheared by turbulent gas stream. Int. J. Multiph. Flow 35 (7), 617-627.

Andrade, J.S., Costa, U.M.S., Almeida, M.P., Makse, H.A., Stanley, H.E., 1999. Inertial effects on fluid flow through disordered porous media. Phys. Rev. Lett. 82, 5249-5252.

Barrenechea, G.R., Le Tallec, P., Valentin, F., 2002. New wall laws for the unsteady incompressible Navier-Stokes equations on rough domains. ESAIM: Math. Modell. Numer. Anal. 36 (2), 177-203.

Basson, A., Gérard-Varet, D., 2008. Wall laws for fluid flows at a boundary with random roughness. Commun. Pure Appl. Math. 61 (7), 941-987.

Bejan, A., Nield, D.A., 2013. Convection in Porous Media. Mechanics of Fluid Flow Through a Porous Medium. Springer New York, New York, NY, pp. 1-29.

Brunazzi, E., Paglianti, A., 1997. Mechanistic pressure drop model for columns containing structured packings. AIChE J. 43 (2), 317-327.

Clavier, R., Chikhi, N., Fichot, F., Quintard, M., 2015. Experimental investigation on single-phase pressure losses in nuclear debris beds: identification of flow regimes and effective diameter. Nucl. Eng. Des. 292, 222-236.

Davit, Y., Quintard, M., 2012. Comment on "Frequency-dependent dispersion in porous media". Phys. Rev. E 86, 1-4.

de Lemos, M.J.S., 2006. In: de Lemos, M.J.S. (Ed.), Turbulence in Porous Media. Elsevier Science Ltd, Oxford.

Dietze, G.F., Ruyer-Quil, C., 2013. Wavy liquid films in interaction with a confined laminar gas flow. J. Fluid Mech. 722, 348-393. 5.

Edwards, D.A., Shapiro, M., Bar-Yoseph, P., Shapira, M., 1990. The influence of reynolds number upon the apparent permeability of spatially periodic arrays of cylinders. Phys. Fluids A 2 (1), 45-55.

Ferrás, L.L., Nóbrega, J.M., Pinho, F.T., 2013. Implementation of slip boundary conditions in the finite volume method: new techniques. Int. J. Numer. Meth. Fluids 72 (7), 724-747.

Firdaouss, M., Guermond, J.L., Le Quéré, P., 1997. Nonlinear corrections to Darcy's law at low reynolds numbers. J. Fluid Mech. 343, 331-350. 7.

Gray, W.G., Majid Hassanizadeh, S., 1998. Macroscale continuum mechanics for multiphase porous-media flow including phases, interfaces, common lines and common points. Adv. Water Resour. 21 (4), 261-281.

Hecht, F., 2013. New development in FreeFem++. J. Numer. Math. 20, 251-266.

Howes, F.A., Whitaker, S., 1985. The spatial averaging theorem revisited. Chem. Eng. Sci. 40 (8), 1387-1392.

Introini, C., Quintard, M., Duval, F., 2011. Effective surface modeling for momentum and heat transfer over rough surfaces: application to a natural convection problem. Int. J. Heat Mass Transf. 54 (15-16), 3622-3641.

Issa, R.I., 1986. Solution of the implicitly discretised fluid flow equations by operator-splitting. J. Comput. Phys. 62 (1), 40-65.

Jin, Y., Uth, M.F., Kuznetsov, A.V., Herwig, H., 2015. Numerical investigation of the possibility of macroscopic turbulence in porous media: a direct numerical simulation study. J. Fluid Mech. 766, 76-103. 3.

Kamrin, K., Bazant, M.Z., Stone, H.A., 2010. Effective slip boundary conditions for arbitrary periodic surfaces: the surface mobility tensor. J. Fluid Mech. 658, 409437.

Koch, D.L., Ladd, A.J.C., 1997. Moderate Reynolds number flows through periodic and random arrays of aligned cylinders. J. Fluid Mech. 349, 31-66.

Lage, J.L., 1998. The fundamental theory of flow through permeable media from Darcy to turbulence. In: Inghamiaon, D.B., Pop, I. (Eds.), Transport Phenomena in Porous Media. Pergamon, Oxford, pp. 1-30.

Lage, J.L., Antohe, B.V., 2000. Darcy's experiments and the deviation to nonlinear flow regime. J. Fluids Eng. 122, 619-625. 
Lage, J.L., Antohe, B.V., Nield, D.A., 1997. Two types of nonlinear pressure-drop versus flow-rate relation observed for saturated porous media. J. Fluids Eng. 119 (3), 700-706.

Lasseux, D., Abbasian Arani, A.A., Ahmadi, A., 2011. On the stationary macroscopic inertial effects for one phase flow in ordered and disordered porous media. Phys. Fluids 23 (7).

Lasseux, D., Valdes Parada, F.J., Ochoa Tapia, J.A., Goyeau, B., 2014. A macroscopic model for slightly compressible gas slip-flow in homogeneous porous media. Phys. Fluids (1994-present) 26 (5).

Mahr, B., Mewes, D., 2007. CFD modelling and calculation of dynamic two-phase flow in columns equipped with structured packing. Chem. Eng. Res. Des. 85 (8), $1112-1122$.

Mahr, B., Mewes, D., 2008. Two-phase flow in structured packings: modeling and calculation on a macroscopic scale. AIChE J. 54 (3), 614-626.

McGlamery, G.G., 1988. Liquid Film Transport Characteristics of Textured Metal Surfaces PhD thesis. University of Texas at Austin.

Nakayama, A., Kuwahara, F., 2008. A general macroscopic turbulence model for flows in packed beds, channels, pipes, and rod bundles. J. Fluids Eng. 130.

Nikou, M.R. Khosravi, Ehsani, M.R., 2008. Turbulence models application on CFD simulation of hydrodynamics, heat and mass transfer in a structured packing. Int. Commun. Heat Mass Transf. 35 (9), 1211-1219.

Olujic, Z., 1999. Effect of column diameter on pressure drop of a corrugated sheet structured packing. Chem. Eng. Res. Des. 77 (6), 505-510.

Owens, S.A., Perkins, M.R., Eldridge, R.B., Schulz, K.W., Ketcham, R.A., 2013. Computational fluid dynamics simulation of structured packing. Ind. Eng. Chem. Res. 52 (5), 2032-2045.

Patankar, S.V., 1980. Numerical Heat Transfer and Fluid Flow. CRC Press, New York, NY, p. 214.

Petre, C.F., Larachi, F., Iliuta, I., Grandjean, B.P.A., 2003. Pressure drop through structured packings: breakdown into the contributing mechanisms by CFD modeling. Chem. Eng. Sci. 58 (1), 163-177.

Quintard, M., Whitaker, S., 1994a. Transport in ordered and disordered porous media II: Generalized volume averaging. Transp. Porous Media 14, 179-206.

Quintard, M., Whitaker, S., 1994b. Transport in ordered and disordered porous media III: Closure and comparison between theory and experiment. Transp. Porous Media 15, 31-49.

Quintard, M., Whitaker, S., 1994c. Transport in ordered and disordered porous media IV: Computer generated porous media for three-dimensional systems. Transp. Porous Media 15, 51-70.
Raynal, L., Royon-Lebeaud, A., 2007. A multi-scale approach for CFD calculations of gas-liquid flow within large size column equipped with structured packing. Chem. Eng. Sci. 62 (24), 7196-7204.

Rocha, J.A., Bravo, J.L., Fair, J.R., 1993. Distillation columns containing structured packings: a comprehensive model for their performance. 1. Hydraulic models. Ind. Eng. Chem. Res. 32 (4), 641-651.

Skjetne, E., Auriault, J.-L., 1999. High-velocity laminar and turbulent flow in porous media. Transp. Porous Media 36 (2), 131-147.

Soulaine, C., Quintard, M., 2014. On the use of a Darcy-Forchheimer like model for a macro-scale description of turbulence in porous media and its application to structured packings. Int. J. Heat Mass Transf. 74 (0), 88-100.

Sternberg, J., 1962. A theory for the viscous sublayer of a turbulent flow. J. Fluid Mech. 13, 241-271. 6.

Trifonov, Y.Y., 2010. Flooding in two-phase counter-current flows: numerical investigation of the gas-liquid wavy interface using the Navier-Stokes equations. Int. J. Multiph. Flow 36 (7), 549-557.

Tsai, R.E., 2010. Mass Transfer Area of Structured Packing PhD thesis. University of Texas, Austin.

Vellingiri, R., Tseluiko, D. Savva, N., Kalliadasis, S., 2013. Dynamics of a liquid film sheared by a co-flowing turbulent gas. Int. J. Multiph. Flow 56 (0), 93-104.

Veran, S., Aspa, Y., Quintard, M., 2009. Effective boundary conditions for rough reactive walls in laminar boundary layers. Int. J. Heat Mass Transf. 52 (15-16), 3712-3725.

Vincent, D., Andro, M., 2002. Ecoulement tangentiel sur une surface rugueuse et loi de navier. Ann. Math. Blaise Pascal 9 (2), 313-327.

Whitaker, S., 1969. Advances in theory of fluid motion in porous media. Ind. Eng. Chem. 61 (12), 14-28.

Whitaker, S., 1986. Flow in porous media I: A theoretical derivation of Darcy's law. Transp. Porous Media 1 (1), 3-25.

Whitaker, S., 1996. The Forchheimer equation: a theoretical development. Transp. Porous Media 25 (1), 27-61.

Whitaker, S., 1999. The Method of Volume Averaging, vol. 13. Springer, Netherlands.

Zami-Pierre, F, de Loubens, R, Quintard, M. Davit, Y, 2016. Transition in the flow of power-law fluids through isotropic porous media. Phys. Rev. Lett. 117, 074502/ $1-074502 / 5$.

Zapke, A., Kröger, D.G., 2000. Countercurrent gas-liquid flow in inclined and vertical ducts - I: Flow patterns, pressure drop characteristics and flooding. Int. J. Multiph. Flow 26 (9), 1439-1455. 